\title{
$N$-heteroatom substitution effect in 3-aza-cope rearrangements
}

\author{
Mário JS Gomes ${ }^{1,2}$, Luis FV Pinto ${ }^{1}$, Paulo MC Glória ${ }^{1}$, Henry S Rzepa ${ }^{3+}$, Sundaresan Prabhakar ${ }^{1+}$ and Ana M Lobo ${ }^{1 *+}$
}

\begin{abstract}
Background: The nature of the heteroatom substitution in the nitrogen of a 3-aza-Cope system is explored.

Results: While N-propargyl isoxazolin-5-ones suffer 3-aza-Cope rearrangements at $60^{\circ} \mathrm{C}$, the corresponding $\mathrm{N}$-propargyl pyrazol-5-ones need a higher temperature of $180^{\circ} \mathrm{C}$ for the equivalent reaction. When the propargyl group is substituted by an allyl group, the temperature of the rearrangement for both type of compounds is less affected by the nature of the heteroatom present. Treatment with a base, such as ethoxide, facilitates the rearrangement, and in the case of isoxazol-5- ones other ring opening reactions take precedence, involving $\mathrm{N}-\mathrm{O}$ ring cleavage of the 5-membered ring. However when base-catalysed decomposition is prevented by substituents, products arising from a room temperature aza-Cope rearrangement are isolated. A possible mechanistic pathway based on free energies derived from density functional calculations involving cyclic intermediates is proposed.
\end{abstract}

Conclusions: The nature of the heteroatom substitution in the nitrogen of a 3-aza-Cope system leads to a remarkable difference in the energy of activation of the reaction.

\section{Background}

Cope-type rearrangements constitute a highly efficient means for carbon-carbon bond formation, offering high regio- and stereocontrol which renders them especially useful in synthesis [1]. The Aza-Cope rearrangement in particular has some advantages over its oxo-counterpart, namely the fine tuning of the reaction temperature which can be achieved by attaching different substituents to the nitrogen of the rearranging system [2-5], by adding charges or by encapsulating it [6]. As previously reported by us appropriately substituted $N$-alkyl(silyl) oxy- $N$-allyl enamines undergo smooth 3,3-sigmatropic rearrangements to the corresponding $N$-alkyl(silyl)oxy imino ethers in good to excellent yields [7]. Theoretical studies also indicate that an oxyanionic substituent on the nitrogen atom reduces substantially the activation energies for these rearrangements [8]. We report in this manuscript the results obtained with the $N$-propargyl and $\mathrm{N}$-allyl 5-membered heterocycles, pyrazolin-5-ones

\footnotetext{
* Correspondence: aml@fct.unl.pt

${ }^{\dagger}$ Equal contributors

'Chemistry Department, REQUIMTE/CQFB, FCT, Universidade Nova de Lisboa, 2829-516, Caparica, Portugal

Full list of author information is available at the end of the article
}

and isoxazolin-5-ones, either under thermolysis or in the presence of a base.

\section{Results and discussion}

The required compounds $\mathbf{1}$ for the rearrangement studies were obtained by alkylation of $\mathbf{3}$ using a Mitsunobu reaction [9] as in Scheme 1. The isolated compounds $\mathbf{1}$ and $\mathbf{2}$ were easily separated by chromatography (cf. Experimental). Heating either $\mathbf{1}$ or $\mathbf{2}$ gave rise to a 3,3sigmatropic rearrangement $[5,10]$ and the same final compound 4 as in Scheme 1 (Table 1, entries 1-4).

In the presence of EtOK and 18-crown-6 ether at r.t., it was found that the propargyl group of 1a isomerized to the corresponding allene $\mathbf{5 a}$ as in Scheme 2, which could in turn suffer upon heating a 3,3-sigmatropic rearrangement leading to $\mathbf{6 a}$. This last compound could also be reached from $2 \mathbf{a}$ by treatment with base to give $7 \mathbf{a}$, followed by thermolysis. Thus coupling of these two reactions opened the way to 3 isomeric compounds of 1a. It is worth noticing that, while the $N$-allenyl 5a (Table 1, entry 5) reacts in a similar way to the $N$-propargyl 1a (entry 1 ) in the 3 -aza-Cope rearrangement, the corresponding $O$-allenyl $7 \mathbf{a}$ (entry 6) and the $O$-propargyl 2a (entry 3) rearrange faster and give better yields. Similar results are found for the pair $\mathbf{1 a} / \mathbf{2 a}$ and
(C) Chemistry Central

(C) 2013 Gomes et al.; licensee Chemistry Central Ltd. This is an Open Access article distributed under the terms of the Creative Commons Attribution License (http://creativecommons.org/licenses/by/2.0), which permits unrestricted use, distribution, and reproduction in any medium, provided the original work is properly cited. 


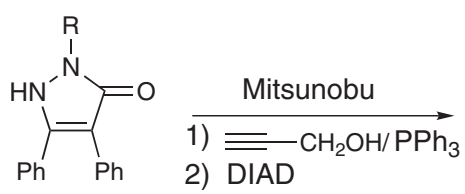

3

a. $R=M e$
b. $R=P h$<smiles></smiles>

1<smiles>[I-]C=[Tl]</smiles>

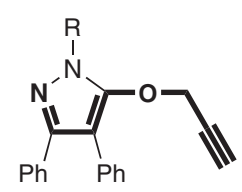

2

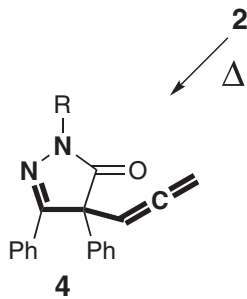

Scheme 1 Synthesis and thermal rearrangement of $\mathrm{N}$-propargyl pyrazolin-5-ones 1 and their isomers 2 .

$\mathbf{1 b} / \mathbf{2 b}$. The reason is probably related to the more facile nature of the 3-oxa-Cope (Claisen) versus the 3-azaCope rearrangements [3].

Next, the $N$-propargyl isoxazolinones $\mathbf{8 a}, \mathbf{b}$ and the $N$-allyl 8c,d [11] were synthesised (cf. Experimental) and heated to give the corresponding rearranged compounds, the allenes 9a,b, (Scheme 3, Table 2, entries 1,2) and the allyl 9c,d (Scheme 4, Table 2, entries 3,4). It was found that the first ones required a much lower temperature for the rearrangement.

The synthesis of $\mathbf{8 e}$ is depicted in Scheme 5 as well as its product after heating (Table 2 , entry 5 ). Desilylation [12] of compound $\mathbf{1 0}$ yielded the corresponding enehydroxylamine 11, which was found to be unstable and to lactonise spontaneously to the 2-allyl-isoxazolone $\mathbf{8 e}$ (Scheme 5). The latter on thermolysis $\left(180^{\circ} \mathrm{C}\right)$ furnished 2-cyano-pent-4-enoic acid ethyl ester 12, as shown. In this case the thermodynamic gain resulting from the loss of carbon dioxide from the rearranged product $9 \mathbf{e}$ is also accompanied by the formation of the cyano derivative 12 [13]. When treated with potassium ethoxide, $8 \mathbf{e}$ led to the formation of $N$-allyl-malonamic acid ethyl ester 13 instead of the rearranged product (Scheme 6).

Table 1 Rearrangements of pyrazolin-5-ones 1 and their isomers 2

\begin{tabular}{|c|c|c|c|}
\hline \# & $\begin{array}{l}\text { Pyrazolinones } 1 \& \\
\text { isomers } 2\end{array}$ & $\begin{array}{l}\text { Conditions temp./ } \\
\text { time }\end{array}$ & $\begin{array}{l}\text { Products yield } \\
(\%)\end{array}$ \\
\hline 1 & 1a: $R=M e$ & $180^{\circ} \mathrm{C} / 60 \mathrm{~m}$ & 4a: $R=\operatorname{Me}(35)$ \\
\hline 2 & 1b: $\mathrm{R}=\mathrm{Ph}$ & $180^{\circ} \mathrm{C} / 10 \mathrm{~m}$ & 4b: $\mathrm{R}=\mathrm{Ph}(72)$ \\
\hline 3 & 2a: $R=M e$ & $180^{\circ} \mathrm{C} / 10 \mathrm{~m}$ & 4a: $R=M e(90)$ \\
\hline 4 & $\mathbf{2 b}: \mathrm{R}=\mathrm{Ph}$ & $180^{\circ} \mathrm{C} / 10 \mathrm{~m}$ & 4b: $\mathrm{R}=\mathrm{Ph}(99)$ \\
\hline 5 & 5a: $R=M e$ & $180^{\circ} \mathrm{C} / 30 \mathrm{~m}$ & $\mathbf{6 a}: R=M e(30)$ \\
\hline 6 & 7a: $R=M e$ & $180^{\circ} \mathrm{C} / 10 \mathrm{~m}$ & $\mathbf{6 a}: R=M e(80)$ \\
\hline
\end{tabular}

asolated yields after flash-chromatography.
Again loss of carbon dioxide, as found earlier by Woodman [14], occurred with a rearrangement possibly involving cyclic intermediates to provide the amide group of 13 [15].The energetics of possible mechanistic pathways for this process were explored using a density functional approach ( $\omega$ B97XD functional, 6-311G(d,p) basis set and SCRF(CPCM) continuum solvation method for ethanol as a model polar solvent). The reaction is sufficiently complex that a significant number of possible mechanistic pathways can be envisaged using the classical "arrow pushing" approach. Here we adopt the approach of incrementally locating pathways to the product and optimizing them for the lowest overall activation free energy. Such an approach of course does not guarantee finding the global energetic minimum in mechanistic pathways. Instead the objective is to find a thermally reasonable pathway that might approximately correspond to the observed rate of the reaction, and along the way eliminating pathways that have unreasonably high activation free energies.

The mechanistic exploration is set out in Scheme 6. The first route explored involved $8 \mathbf{e}$ and ethoxide anion acting as a nucleophile. Addition of the ethoxide gives $\mathbf{8 e - 1}$ as the first intermediate, followed by ring closure to $\mathbf{8 e - 2}$ [16]. The most direct route (Occams's razor) to 13 is by a dyotropic rearrangement of $8 \mathbf{e}-\mathbf{2}$ to $8 \mathbf{e}-\mathbf{3}$, followed by protonation and decarboxylation. The energetic high point of this pathway is the transition state for the dyotropic rearrangement of the intermediate oxaziridine (8e-TS1) which manifests as unreasonably high (Additional file 1: Table S1, entry 4) ${ }^{\text {a }}$.

The next route explored was with ethoxide acting as a base, abstracting the allylic proton to give the intermediate 8e-4. A ring closure to $\mathbf{8 e - 5}$, followed by 1,3 intramolecular proton transfer to give $\mathbf{8 e - 6}$, a second proton transfer to reform alkoxide anion and final ring opening to $\mathbf{8 e - 7}$ gives $\mathbf{8 e - 8}$, which is merely a tautomer of $\mathbf{8 e - 3}$ and can decarboxylate as before to give 13. The 


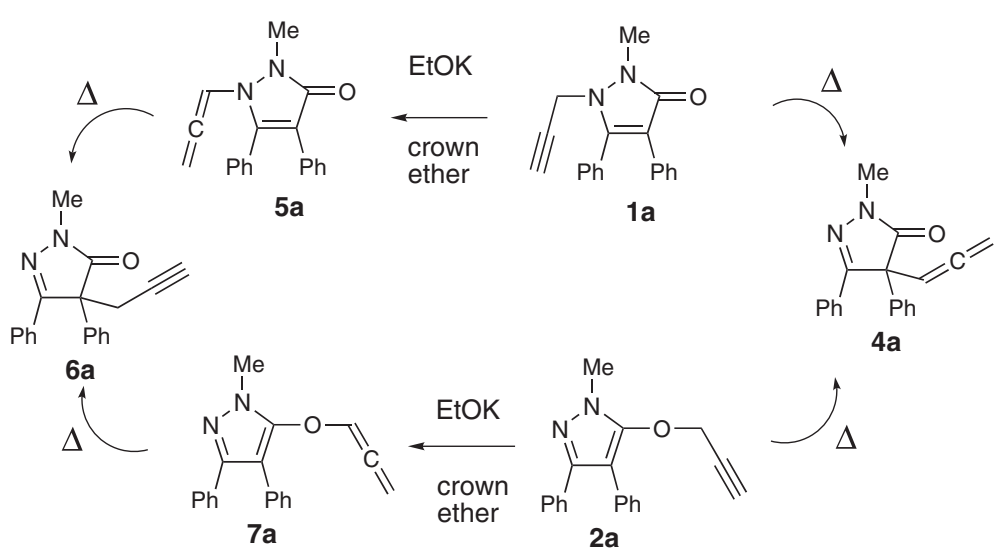

Scheme 2 Thermal and base catalyzed rearrangements of pyrazolin-5-ones and their isomers.

high point of this pathway is 8e-TS2, which shows as an entirely reasonable barrier for the reaction (Additional file 1: Table S1, entry 5). Whilst this does not constitute a formal mechanistic proof of the reaction, it does imply a reasonable one, and further that any alternative mechanism must have an overall lower energy barrier than this one.

Whereas it is traditional, indeed conventional, for the mechanism of a synthetic pathway to be speculated upon using mechanistic reaction arrows, we suggest here that increasingly such speculations must be supported by a computational exploration of the potential free energy surface. In this case, this has been done with a procedure which, including as it does dispersion corrections for all species, a triple-zeta quality basis with polarization functions, and a correction for continuum solvation, is suggested to be the minimum in quality appropriate for such exploration.

Exposure of 2-allyl-3,4-diphenyl-isoxazolin-5-one (8d) to similar conditions gave rise after 10 minutes to $\mathbf{1 4}$ in $25 \%$ yield (along with desoxybenzoin in $45 \%$, Scheme 7 ) with no 3-aza-Cope rearrangement being observed. Compound 14 was found to generate desoxybenzoin (15 $\mathbf{R}=\mathbf{H}$ ) by quenching under aqueous acidic conditions. Quenching the reaction with deuterated acetic acid $\left(\mathrm{CH}_{3} \mathrm{COOD}\right)$ resulted in the isolation of monodeuterated

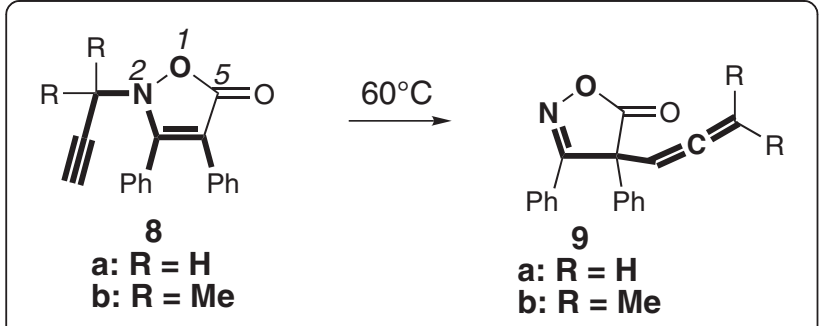

Scheme 3 3-Aza-Cope rearrangement of $\mathrm{N}$-propargyl isoxazolin-5-ones $8 \mathrm{a}, \mathrm{b}$. benzoin $(\mathbf{1 5} \mathbf{R}=\mathbf{D})$. A possible mechanism is shown and follows along equivalent intermediates, $\mathbf{8 d - 3}$ or the oxaziridine derived 8d-4 [17], until reaching 8d-5. A phenyl substituent on carbon 3 in the $\beta$-lactone ring of 8d-5 now leads instead to $\mathbf{1 4}$.

Substrate $\mathbf{8 b}$, where formation of the carbanion adjacent to the ring nitrogen of the oxazolidinone is blocked by substitution, was next treated with potassium ethoxide $(0.1 \mathrm{eq})$, in the presence of 18 -crown- 6 ether, at room temperature (5 days), and gave rise to $9 \mathbf{b}$ in $55 \%$ yield (cf. Scheme 8). Here the opening of the five membered ring leading to $\mathbf{8 b - 1}$ might lower the energy of the reaction either by allowing an easier access to the conformation leading to the transition state or by anionic charge acceleration of the rearrangement. The importance of EtOK was noticed further when, after 5 days at r. t., in its absence, the rearrangement of $\mathbf{8 b}$ was observed to occur, but in a much lower yield $(<10 \%)$. The same compound treated with LDA or tert-BuLi, in the

Table 2 Thermolysis of isoxazolin-5-ones 8 in the presence and absence of potassium ethoxide

\begin{tabular}{|c|c|c|c|c|}
\hline \multirow[t]{2}{*}{ \# } & \multirow[t]{2}{*}{ Compounds 8} & \multirow{2}{*}{$\begin{array}{l}\text { Conditions } \\
\text { Temp./Time }\end{array}$} & \multicolumn{2}{|c|}{ Products Yield $^{\mathrm{a}}(\%)$} \\
\hline & & & 9 & Others \\
\hline 1 & 8a: $R=H$ & $60^{\circ} \mathrm{C} / 30 \mathrm{~m}$ & $9 a(75)$ & - \\
\hline 2 & $\mathbf{8 b}: R=M e$ & $60^{\circ} \mathrm{C} / 10 \mathrm{~m}$ & $9 \mathbf{b}(85)$ & - \\
\hline 3 & 8c: $R^{\prime}=M e$ & $180^{\circ} \mathrm{C} / 7 \mathrm{~h}$ & $9 c(95)^{b}$ & - \\
\hline 4 & 8d: $R^{\prime}=P h$ & $180^{\circ} \mathrm{C} / 5 \mathrm{~h}$ & 9d (97) & \\
\hline 5 & $8 e$ & $180^{\circ} \mathrm{C} / 5 \mathrm{~h}$ & - & $12(80)$ \\
\hline 6 & $8 e$ & $\mathrm{EtOK}^{\mathrm{c}, \mathrm{d}} / \mathrm{RT} / 5 \mathrm{~h}$ & - & $13(80)$ \\
\hline 7 & $\mathbf{8 d}: R^{\prime}=P h$ & $\mathrm{EtOK}^{\mathrm{c}, \mathrm{d}} / \mathrm{RT} / 10 \mathrm{~m}$ & - & $14(25) 15(45)$ \\
\hline \multirow[t]{2}{*}{8} & $\mathbf{8 b}: R=M e$ & $\mathrm{EtOK}^{\mathrm{c}, \mathrm{e}} / \mathrm{RT} / \mathrm{d} \mathrm{d}$ & $\mathbf{9 b}(55)$ & - \\
\hline & & $\mathrm{EtOK}^{\mathrm{c}, \mathrm{d}} / \mathrm{RT} / 10 \mathrm{~m}$ & 9b (30) & - \\
\hline
\end{tabular}

asolated yields after flash-chromatography. ${ }^{\mathrm{b}} \mathrm{See}$ ref. 11. ${ }^{\mathrm{c}}$ In the presence of 18-crown-6 ether. ${ }^{\mathrm{d}}$ In the presence of EtOK (1.5 eq). ${ }^{\mathrm{e}}$ In the presence of EtOK (0.1 eq). 


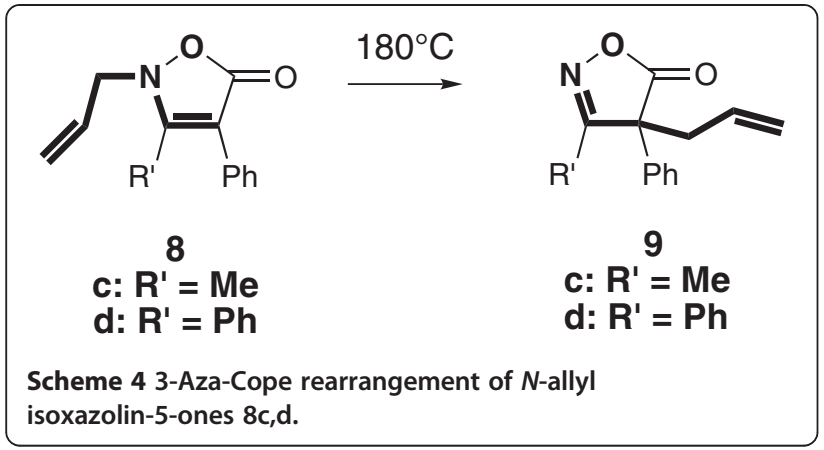

presence of 12-crown- 6 ether and in the same conditions of concentration and temperature, showed no reaction. Thus the anion derived from the possible base removal of the terminal triple bond proton does not appear to accelerate the rearrangement, but the oxyanion formed upon the isoxazolidinone ring opening did have a moderately positive effect enabling the rearrangement to occur at room temperature.

\section{Conclusions}

In conclusion: 1) substitution of the nitrogen-1 of pyrazolin-5-ones by oxygen lowers the temperature of the 3-aza-Cope rearrangement of isoxazolin-5-ones vis-à-vis the corresponding pyrazolin-5-ones when the rearranging element includes a propargyl group attached to the heterocyclic nitrogen-2; 2) when the propargyl group is replaced by an allyl group the rearranging temperature is higher and similar in both compounds; 3) treatment with potassium ethoxide as base in the presence of 12-crown- 6 ether at room temperature, while leaving the pyrazolin5-one heterocycle untouched, leads to ring opening of

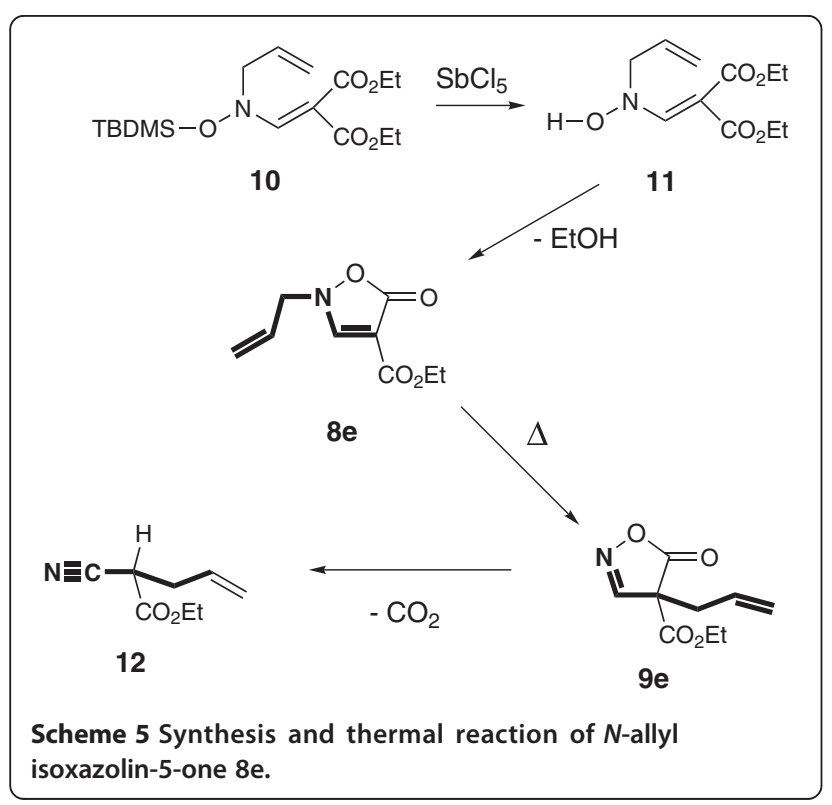

isoxazolin-5-ones, followed by further reactions. The putative oxaziridine or other cyclic intermediate then suffer base catalysed ring opening reaction whenever there are protons available in the carbon $\alpha$ to the ring nitrogen, leading to a 6-membered aza,oxa-ring system. When such position is blocked by substituents the 3-aza-Cope rearrangement occurs at room temperature.

\section{Experimental}

\section{General}

Melting points were determined on a Reichert Thermovar apparatus and are uncorrected. Ordinary mass spectra were recorded on a Fisons TRIO 2000 or AEI MS-9 spectrometer. High-resolution MS spectra (HRMS) were obtained on a FT- ICR/ MS Finnigan FT/MS 2001-DT spectrometer at $70 \mathrm{eV}$ by electron impact or on a Finnigan MAT 900 ST spectrometer by ESI. Infrared (IR) spectra were recorded on a Perkin-Elmer 1000X FT-IR spectrometer. Proton and ${ }^{13} \mathrm{C}$ NMR spectra were recorded in $\mathrm{CDCl}_{3}$ on a Bruker ARX 400 spectrometer $(400 \mathrm{MHz}$ for ${ }^{1} \mathrm{H}, 100.63 \mathrm{MHz}$ for ${ }^{13} \mathrm{C}$ ). Chemical shifts are reported relative to tetramethylsilane as the internal reference $\left(\delta_{\mathrm{H}} 0.00\right)$ for ${ }^{1} \mathrm{H}$ NMR spectra and to $\mathrm{CDCl}_{3}\left(\delta_{\mathrm{C}} 77.00\right)$ for ${ }^{13} \mathrm{C}$ NMR spectra. IR spectra were run on an FT PerkinElmer 1000 instrument, with absorption frequencies expressed in reciprocal centimeters. Thin-layer chromatography was performed on Merck silica gel $60 \mathrm{~F}_{254} 0.2 \mathrm{~mm}$ thick plates, visualized under UV light or by exposing to iodine vapour. For preparative separations the plates were $0.5-1 \mathrm{~mm}$ thick. For flash chromatography silica Merck Kieselgel 60, 70-230 mesh was used. Usual work-up implies drying the water- or brine-washed organic extracts over anhydrous sodium sulfate or magnesium sulfate, followed by filtration and solvent removal under reduced pressure. Anhydrous solvents were dried and freshly distilled by standard methods [18].

\section{Synthesis of starting materials} Ethyl 3-oxo-2-phenyl-butanoate

To a solution of ethyl acetoacetate $(1.5 \mathrm{~g}, 11.5 \mathrm{mmol})$ in $\mathrm{CH}_{2} \mathrm{Cl}_{2}(45 \mathrm{~mL})$, was added triphenylbismuth carbonate [19] (6.33 g, $12.6 \mathrm{mmol})$. After being stirred under argon for $24 \mathrm{~h}$ at $40^{\circ} \mathrm{C}$, the mixture was filtered and concentrated at reduced pressure. The crude was purified by $\mathrm{CC}$ (silica; $\mathrm{Et}_{2} \mathrm{O} / \mathrm{n}$-hexane 1:3) to give the title compound as a yellow oil [20] (1.44 g, 61\%): IR ( NaCl) $v_{\max }\left(\mathrm{cm}^{-1}\right): 3063,2983,1745(\mathrm{C}=\mathrm{O}$, ester $), 1721(\mathrm{C}=$ $\mathrm{O}$, ketone). ${ }^{1} \mathrm{H}$ NMR (400 $\mathrm{MHz} \mathrm{CDCl}_{3}$ ) keto-enol equilibrium, enol (55\%): $\delta 1.18\left(3 \mathrm{H}, \mathrm{t}, J=7.2 \mathrm{~Hz}, \mathrm{OCH}_{2} \mathrm{CH}_{3}\right)$, $1.85\left(3 \mathrm{H}, \mathrm{s}, \mathrm{CH}_{3} \mathrm{CO}\right), 4.23\left(2 \mathrm{H}, \mathrm{m}, \mathrm{OCH}_{2} \mathrm{CH}_{3}\right), 7.14-$ $7.38(5 \mathrm{H}, \mathrm{m}, \mathrm{Ar}-\mathrm{H}), 13.13(1 \mathrm{H}, \mathrm{s}, \mathrm{OH})$; ketone $(45 \%)$; $1.27\left(3 \mathrm{H}, \mathrm{t}, J=7.2 \mathrm{~Hz}, \mathrm{OCH}_{2} \mathrm{CH}_{3}\right), 2.18\left(3 \mathrm{H}, \mathrm{s}, \mathrm{CH}_{3} \mathrm{CO}\right)$, $4.23\left(2 \mathrm{H}, \mathrm{m}, \mathrm{OCH}_{2} \mathrm{CH}_{3}\right), 4.69(1 \mathrm{H}, \mathrm{s}, \mathrm{PhCH}), 7.14-$ $7.38(5 \mathrm{H}, \mathrm{m}, \mathrm{Ar}-\mathrm{H})$. 


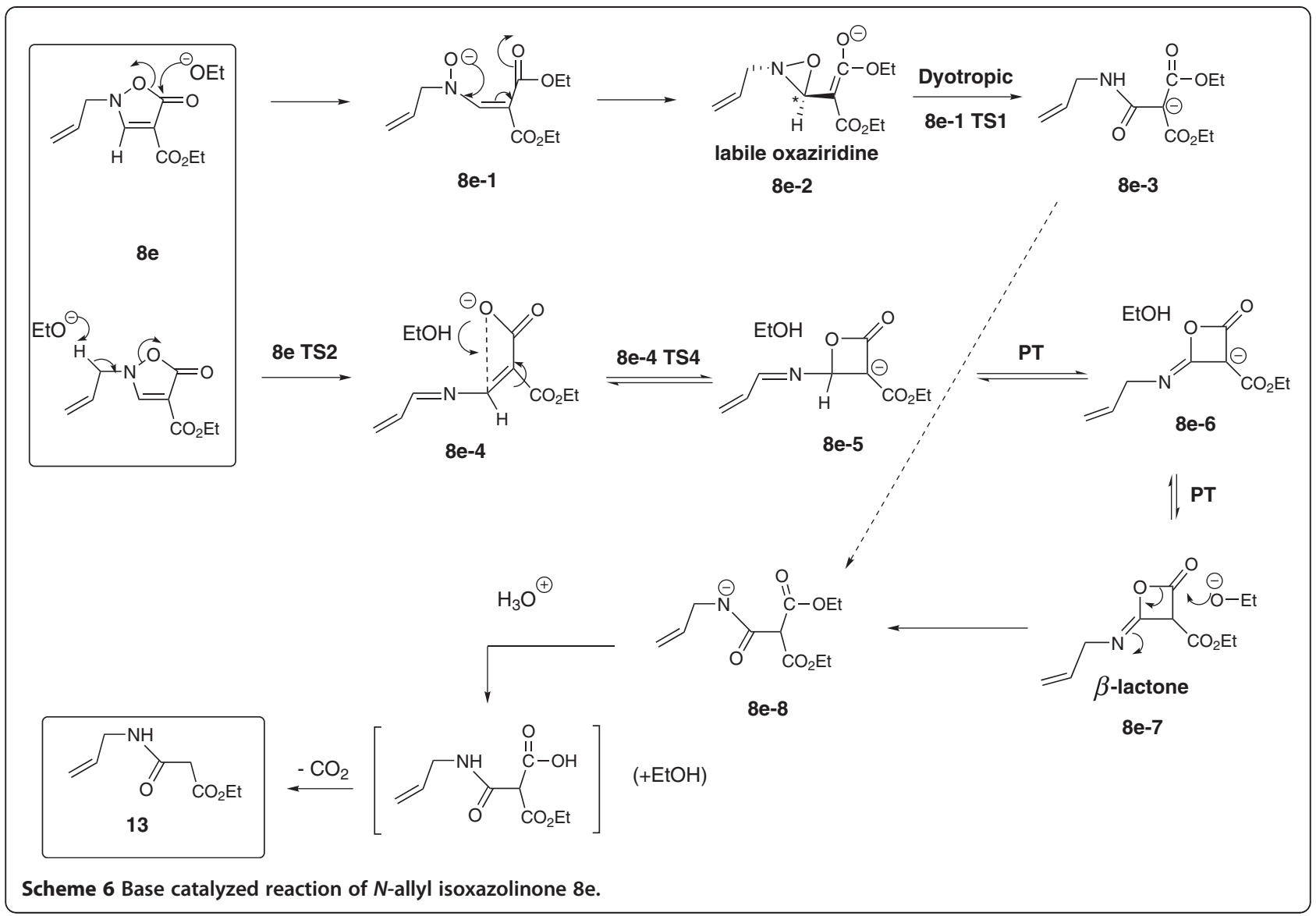

\section{Ethyl 3-oxo-2,3-diphenyl-propanoate}

To a solution of ethyl 3-oxo-3-phenyl-propionate (1.48 g, $7.7 \mathrm{mmol})$ in $\mathrm{CH}_{2} \mathrm{Cl}_{2}(25 \mathrm{~mL})$, was added triphenylbismuth carbonate $(4.22 \mathrm{~g}, 8.5 \mathrm{mmol})$. After being stirred under argon for $24 \mathrm{~h}$ at $40^{\circ} \mathrm{C}$, the mixture was filtered and concentrated at reduced pressure. The crude was purified by $\mathrm{CC}$ (silica; $\mathrm{Et}_{2} \mathrm{O} / n$-hexane 1:3) to give a viscous oil. Recrystallization from ethanol yielded the title compound (1.34 g, 65\%): $\mathrm{mp} 88-89^{\circ} \mathrm{C}$ (ethanol) (lit.: [21] mp 89-90 ${ }^{\circ} \mathrm{C}$ ). IR $(\mathrm{KBr}) v_{\max }\left(\mathrm{cm}^{-1}\right): 3063,2985,1741(\mathrm{C}=\mathrm{O}$, ester $), 1674$ $\left(\mathrm{C}=\mathrm{O}\right.$, ketone). ${ }^{1} \mathrm{H}$ NMR $\left(400 \mathrm{MHz} \mathrm{CDCl}_{3}\right)$ keto-enol equilibrium, enol (12\%): $\delta 1.25\left(3 \mathrm{H}, \mathrm{t}, J=7.2 \mathrm{~Hz}, \mathrm{OCH}_{2} \mathrm{CH}_{3}\right)$, $4.23\left(2 \mathrm{H}, \mathrm{m}, \mathrm{OCH}_{2} \mathrm{CH}_{3}\right), 7.11-7.99(10 \mathrm{H}, \mathrm{m}, \mathrm{Ar}-\mathrm{H})$, $13.66(1 \mathrm{H}, \mathrm{s}, \mathrm{OH})$, and ketone (88\%): $\delta 1.25(3 \mathrm{H}, \mathrm{t}, J=7.2$ $\left.\mathrm{Hz}, \mathrm{OCH}_{2} \mathrm{CH}_{3}\right), 4.23\left(2 \mathrm{H}, \mathrm{m}, \mathrm{OCH}_{2} \mathrm{CH}_{3}\right), 5.62(1 \mathrm{H}, \mathrm{s}$, $\mathrm{PhCH}), 7.11$ - 7.99 (10 H, m, Ar-H).

\section{Methyl-4-phenylisoxazolin-5-one}

To a solution of ethyl 3-oxo-2-phenyl-butanoate $(50 \mathrm{mg}$, $0.24 \mathrm{mmol})$ in $\mathrm{EtOH}(0.5 \mathrm{~mL})$ and $\mathrm{H}_{2} \mathrm{O}(0.1 \mathrm{~mL})$, was added hydroxylamine hydrochloride $(32 \mathrm{mg}, 0.46 \mathrm{mmol}$ ) and sodium acetate $(6.4 \mathrm{mg}, 0.078 \mathrm{mmol})$. The solution was refluxed with continuous stirring under argon for $3 \mathrm{~h}$,
$37 \% \mathrm{HCl}(32 \mu \mathrm{L})$ was added and the mixture refluxed for further $30 \mathrm{~min}$. The solvent was removed under reduced pressure and the crude diluted with distilled water $(2 \mathrm{~mL})$ and extracted with $\mathrm{Et}_{2} \mathrm{O}(3 \times 5 \mathrm{~mL})$. The organic extract was dried, filtered and concentrated under reduced pressure. Purification by CC (silica; AcOEt) yielded the title compound (32 mg, 66\%): $\mathrm{mp} 138-140^{\circ} \mathrm{C}$ (ethyl acetate) (lit.: [22] $\left.\mathrm{mp} 139-140^{\circ} \mathrm{C}\right)$. IR ( $\left.\mathrm{KBr}\right) v_{\max }\left(\mathrm{cm}^{-1}\right): 3322$ (NH), 1669 (C=O). ${ }^{1} \mathrm{H}$ NMR (400 $\left.\mathrm{MHz} \mathrm{CDCl}_{3}\right)$ two distinct forms, isoxazol-5 $(2 \mathrm{H})$-one $(\mathrm{A})$ and isoxazole-5 $(4 \mathrm{H})$ one (B) $(\mathbf{A}: \mathbf{B}=90: 10) \mathbf{A}, \delta 2.35\left(3 \mathrm{H}, \mathrm{s}, \mathrm{CH}_{3}\right), 6.24(1 \mathrm{H}, \mathrm{sl}$, $\mathrm{NH}), 7.14-7.56(5 \mathrm{H}, \mathrm{m}, \mathrm{Ar}-\mathrm{H})$; $\mathbf{B}, \delta 2.04\left(3 \mathrm{H}, \mathrm{s}, \mathrm{CH}_{3}\right)$, $4.40(1 \mathrm{H}, \mathrm{s}, \mathrm{CH}), 7.14-7.56(5 \mathrm{H}, \mathrm{m}, \mathrm{Ar}-\mathrm{H})$.

\section{Diphenylisoxazolin-5-one}

To a solution of ethyl 3-oxo-2,3-diphenyl-propanoate (65 mg, $0.27 \mathrm{mmol})$ in EtOH $(0.5 \mathrm{~mL})$ and $\mathrm{H}_{2} \mathrm{O}(0.1 \mathrm{~mL})$, was added hydroxylamine hydrochloride $(32 \mathrm{mg}$, $0.46 \mathrm{mmol})$ and sodium acetate $(6.4 \mathrm{mg}, 0.078 \mathrm{mmol})$. The solution was refluxed with continuous stirring under argon for $3 \mathrm{~h}$ and processed as in the previous reaction to yield the title compound (40 $\mathrm{mg}, 61 \%)$ as a white powder: $\mathrm{mp}: 159^{\circ} \mathrm{C}$ (dec.) (benzene) (lit.: [23] mp 


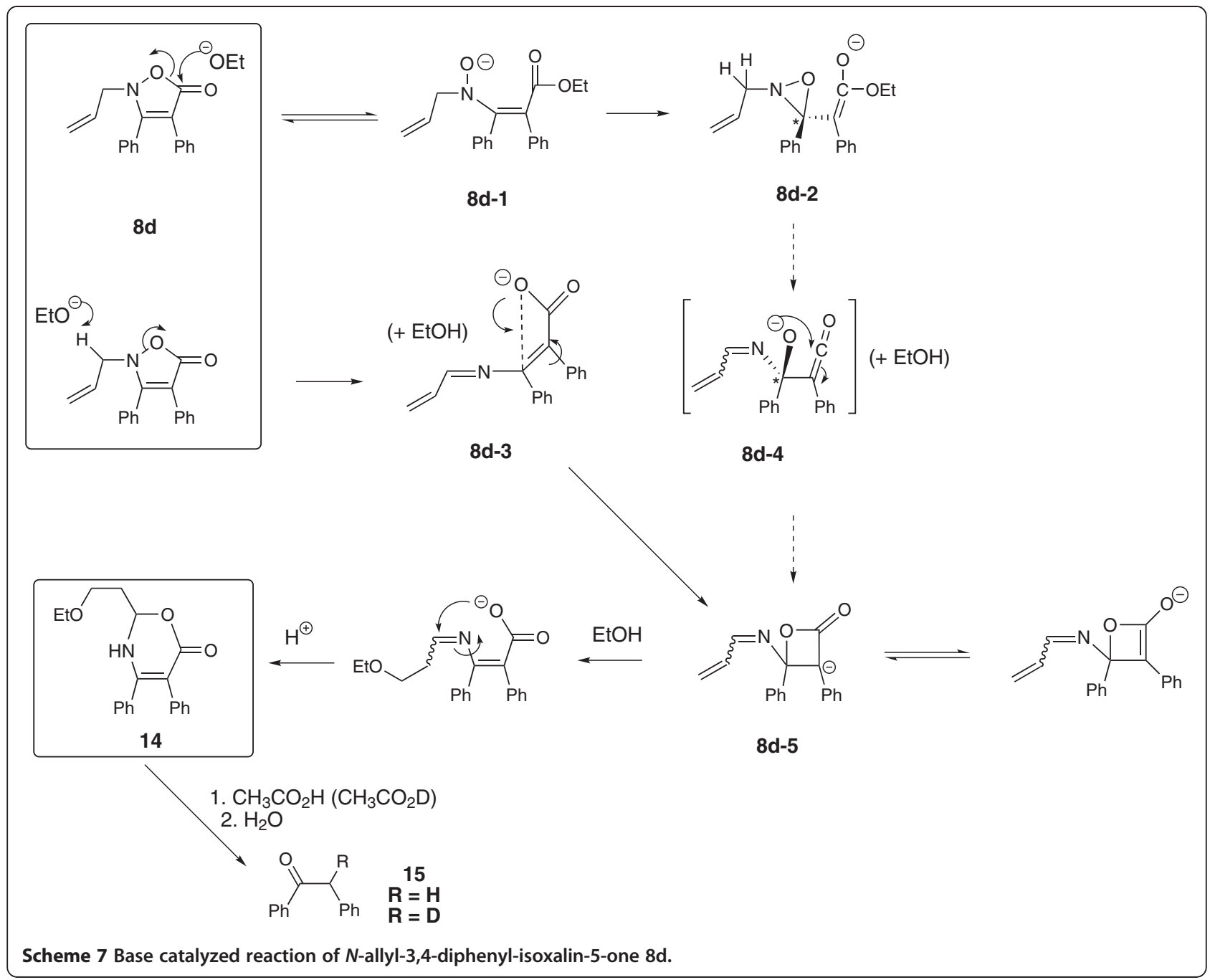

$\left.159^{\circ} \mathrm{C}\right)$. IR $(\mathrm{KBr}) v_{\max }\left(\mathrm{cm}^{-1}\right): 3297(\mathrm{NH}), 1680(\mathrm{C}=\mathrm{O})$. ${ }^{1} \mathrm{H}$ NMR $\left(400 \mathrm{MHz} \mathrm{CDCl}_{3}\right) \delta 5.86(1 \mathrm{H}, \mathrm{sl}, \mathrm{NH}), 7.01-$ $7.79(10 \mathrm{H}, \mathrm{m}, \mathrm{Ar}-\mathrm{H})$.

\section{General procedure for the synthesis of pyrazolinones}

To a stirred ethanolic solution $(\approx 0.5 \mathrm{M})$ of ethyl 3-oxo2,3-diphenylpropanoate [21] (1 eq.) in ethanol is added the desired hydrazine (1 eq.) and the reaction heated to reflux under argon. After total consumption of both starting materials (TLC control: silica, $\mathrm{CH}_{2} \mathrm{Cl}_{2}: \mathrm{MeOH}, 9: 1$ ), the solvent is evaporated under reduced pressure and the product is purified by hot recrystallization using AcOEt.

\section{Diphenyl-1-methyl-2-pyrazolin-5-one (3a)}

From methyl hydrazine, compound 3a obtained after $18 \mathrm{~h}$ as colourless crystals (50\%): mp: $214-216^{\circ} \mathrm{C}$ (AcOEt). ${ }^{1} \mathrm{H}$ NMR (400 MHz $\left.\mathrm{CDCl}_{3}\right) \delta 7.72-7.20(10 \mathrm{H}, \mathrm{m}, \mathrm{Ar}-\mathrm{H})$, $3.70\left(3 \mathrm{H}, \mathrm{s}, \mathrm{NCH}_{3}\right) .{ }^{13} \mathrm{C} \mathrm{NMR}\left(100.62 \mathrm{MHz} \mathrm{CDCl}_{3}\right) \delta 36.4$ $\left(\mathrm{NCH}_{3}\right), 108.4,124.9,126.9,127.2,129.0,131.8,145.3$,
$165.8(\mathrm{C}=\mathrm{O})$. IR $\left(\mathrm{KBr} ; \mathrm{cm}^{-1}\right): 3235(\mathrm{~N}-\mathrm{H}), 1695(\mathrm{C}=\mathrm{O})$. EIMS (m/z, \%): 250 ( $\left.\mathrm{M}^{+}, 100\right), 178$ (58), 77 (26). HRMS: calcd for $\mathrm{C}_{16} \mathrm{H}_{14} \mathrm{~N}_{2} \mathrm{O}$ : 250.11076; found: 250.11061 .

\section{Triphenyl-2-pyrazolin-5-one (3b)}

From phenylhydrazine, compound $3 \mathbf{b}$ obtained after $24 \mathrm{~h}$ as colourless crystals (71\%): mp $198-199^{\circ} \mathrm{C}$ (AcOEt). ${ }^{1} \mathrm{H}$ NMR (400 MHz DMSO-d $\left./ \mathrm{CDCl}_{3}, 1: 2\right) \delta 10.97(1 \mathrm{H}, \mathrm{bs}$, $\mathrm{OH}), 7.84-7.25(15 \mathrm{H}, \mathrm{m}, \mathrm{Ar}-\mathrm{H})$. IR $\left(\mathrm{KBr}, \mathrm{cm}^{-1}\right): 3236$ $(\mathrm{N}-\mathrm{H}), 1697(\mathrm{C}=\mathrm{O})$. EIMS $(\mathrm{m} / \mathrm{z}, \%): 312\left(\mathrm{M}^{+}, 78\right), 178$ (66), 77 (100). HRMS: calcd for $\mathrm{C}_{21} \mathrm{H}_{16} \mathrm{~N}_{2} \mathrm{O}$ : 312.12626; found: 312.12661 .

\section{Mitsunobu propargylation of pyrazolinones 3}

General procedure: To the desired pyrazolone (3a or $\mathbf{3 b}$ ) (1.0 eq.) dissolved in dry THF $(\approx 0.15 \mathrm{M})$ under argon, $\mathrm{PPh}_{3}$ (1.2 eq.) and the required propargyl alcohol (1.0 eq.) are added. The reaction is cooled to $0^{\circ} \mathrm{C}$ and DIAD (1.2 eq.) in dry THF $(\approx 1.5 \mathrm{M})$ is added dropwise. The 


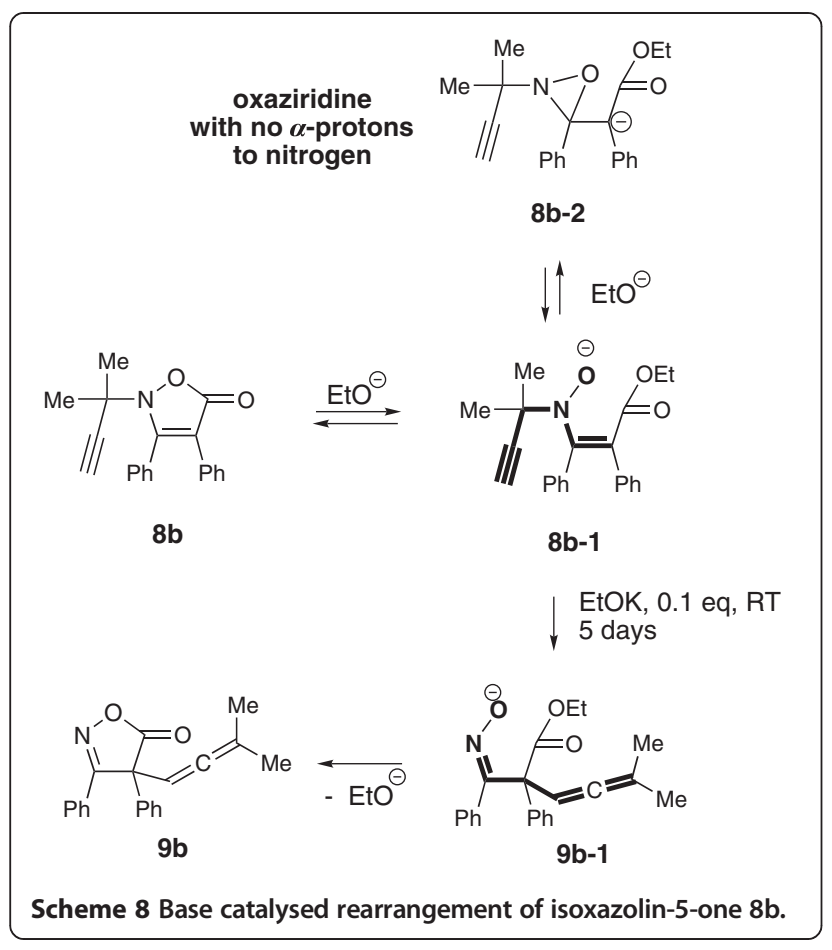

reaction is allowed to reach room temperature and after the reaction is finished (tlc control), the solvent is removed under vacuum. The products are purified by flash chromatography [AcOEt $/ n$-hexane, $(1: 1) \rightarrow$ AcOEt]. Following the general procedure, compounds (1a) and (2a) were obtained from $\mathbf{3 a}$, and $\mathbf{1 b}$ and $\mathbf{2 b}$ from $\mathbf{3 b}$.

\section{Diphenyl-1-methyl-2-propargylpyrazolin-5-one (1a)}

(65\%), colourless crystals, mp $123-124^{\circ} \mathrm{C}$ (AcOEt). ${ }^{1} \mathrm{H}$ NMR (400 MHz $\left.\mathrm{CDCl}_{3}\right) \delta 7.47-7.34(6 \mathrm{H}, \mathrm{m}, \mathrm{Ar}-\mathrm{H})$, $7.24-7.15(4 \mathrm{H}, \mathrm{m}, \mathrm{Ar}-\mathrm{H}), 4.14(2 \mathrm{H}, \mathrm{d}, J=2.0 \mathrm{~Hz}$, $\left.\mathrm{NCH}_{2} \mathrm{C} \equiv \mathrm{CH}\right), 3.49\left(3 \mathrm{H}, \mathrm{s}, \mathrm{NCH}_{3}\right), 2.21(1 \mathrm{H}, \mathrm{t}, J=2.0$ $\left.\mathrm{Hz}, \mathrm{NCH}_{2} \mathrm{C} \equiv \mathrm{CH}\right) .{ }^{13} \mathrm{C} \mathrm{NMR}\left(100.62 \mathrm{MHz} \mathrm{CDCl}_{3}\right) \delta$ $165.5(\mathrm{C}=\mathrm{O}), 152.7[\mathrm{NC}(\mathrm{Ph})], 130.4,130.0,129.4$, 129.0, 129.0, 128.7, 127.9, 126.7, 115.2, 75.4, 74.5, 39.0, 29.2. I.R. $\left(\mathrm{NaCl} ; \mathrm{cm}^{-1}\right)$ : 3279, 2109, $1651(\mathrm{C}=\mathrm{O})$. EIMS (m/z, \%): $288\left(\mathrm{M}^{+}, 46\right), 277$ (100), $249\left(\mathrm{C}_{16} \mathrm{H}_{13} \mathrm{~N}_{2} \mathrm{O}^{+}\right.$, 25), 199 (32); 77 (45). HRMS: calcd for $\mathrm{C}_{19} \mathrm{H}_{16} \mathrm{~N}_{2} \mathrm{O}$ : 288.126263; found: 288.126523.

\section{3,4-Diphenyl-1-methyl-5-propargyloxy-pyrazole (2a)} (35\%), colourless oil. ${ }^{1} \mathrm{H}$ NMR (400 $\left.\mathrm{MHz} \mathrm{CDCl}_{3}\right) \delta 7.46-$ $7.43(2 \mathrm{H}, \mathrm{m}, \mathrm{Ar}-\mathrm{H}), 7.34-7.22(8 \mathrm{H}, \mathrm{m}, \mathrm{Ar}-\mathrm{H}), 4.40$ $\left(2 \mathrm{H}, \mathrm{d}, J=2.4 \mathrm{~Hz}, \mathrm{OCH}_{2} \mathrm{C} \equiv \mathrm{CH}\right), 3.86\left(3 \mathrm{H}, \mathrm{s}, \mathrm{NCH}_{3}\right)$, $2.49\left(1 \mathrm{H}, \mathrm{t}, J=2.4 \mathrm{~Hz}, \mathrm{OCH}_{2} \mathrm{C} \equiv \mathrm{CH}\right) .{ }^{13} \mathrm{C} \mathrm{NMR}(100.62$ $\left.\mathrm{MHz}^{\mathrm{CDCl}} \mathrm{CD}_{3}\right) \delta 149.8[\mathrm{~N}=\mathrm{C}(\mathrm{Ph})], 147.5[\mathrm{~N}=\mathrm{C}(\mathrm{Ph}) \mathrm{C}$ $(\mathrm{Ph})=\mathrm{C}], 133.7(\mathrm{Ar}), 132.0(\mathrm{Ar}), 129.6(\mathrm{Ar}), 128.5(\mathrm{Ar})$, 128.1 (Ar), 127.8 (Ar), 127.4 (Ar), 126.8 (Ar), 105.6 [N = $\mathrm{C}(\mathrm{Ph}) \mathrm{C}(\mathrm{Ph})=\mathrm{C}], 77.7\left(\mathrm{OCH}_{2} \mathrm{C} \equiv \mathrm{CH}\right), 76.7\left(\mathrm{OCH}_{2} \mathrm{C} \equiv\right.$
$\mathrm{CH}), \quad 61.0\left(\mathrm{OCH}_{2} \mathrm{C} \equiv \mathrm{CH}\right), \quad 34.6\left(\mathrm{NCH}_{3}\right)$. I.R. $(\mathrm{NaCl}$, $\left.\mathrm{cm}^{-1}\right): 3289,2123$. EIMS (m/z, \%): $288\left(\mathrm{M}^{+}, 40\right), 249$ $\left(\mathrm{C}_{16} \mathrm{H}_{13} \mathrm{~N}_{2} \mathrm{O}^{+}, 100\right), 221$ (18), 178 (5), 115 (25), 91 (20). HRMS: calcd for $\mathrm{C}_{19} \mathrm{H}_{16} \mathrm{~N}_{2} \mathrm{O}$ : 288.126263; found: 288.126219.

\section{2-Propargyl-1,3,4-triphenyl-2-pyrazolin-5-one (1b)}

(35\%), yellowish oil. ${ }^{1} \mathrm{H} \mathrm{NMR}\left(400 \mathrm{MHz} \mathrm{CDCl}_{3}\right) \delta 7.65$ $(2 \mathrm{H}, \mathrm{d}, J=7.6 \mathrm{~Hz}, \mathrm{Ar}-\mathrm{H}), 7.52-7.41(8 \mathrm{H}, \mathrm{m}, \mathrm{Ar}-\mathrm{H})$, $7.33-7.19(5 \mathrm{H}, \mathrm{m}, \mathrm{Ar}-\mathrm{H}), 4.03(2 \mathrm{H}, \mathrm{d}, J=2.2 \mathrm{~Hz}$, $\left.\mathrm{NCH}_{2} \mathrm{C} \equiv \mathrm{CH}\right), 2.16\left(1 \mathrm{H}, \mathrm{t}, J=2.2 \mathrm{~Hz}, \mathrm{NCH}_{2} \mathrm{C} \equiv \mathrm{CH}\right) .{ }^{13} \mathrm{C}$ NMR (100.62 MHz CDCl $\left.\mathrm{MH}_{3}\right) \delta 464.6(\mathrm{C}=\mathrm{O}), 154.9$ [NC $(\mathrm{Ph})=\mathrm{C}(\mathrm{Ph})], 134.8(\mathrm{Ar}), 130.2(\mathrm{Ar}), 130.1(\mathrm{Ar}), 129.5$ (Ar), 129.2 (Ar), 129.0 (Ar), 129.0 (Ar), 128.0 (Ar), 127.0 (Ar), 126.6 (Ar), $123.5(\mathrm{Ar}), 116.6[\mathrm{NC}(\mathrm{Ph})=C(\mathrm{Ph})], 74.6$ $\left(\mathrm{NCH}_{2} \mathrm{C} \equiv \mathrm{CH}\right), 74.1\left(\mathrm{NCH}_{2} \mathrm{C} \equiv \mathrm{CH}\right), 40.6\left(\mathrm{NCH}_{2} \mathrm{C} \equiv \mathrm{CH}\right)$. IR (film, $\mathrm{cm}^{-1}$ ): 3298, 2116, $1667(\mathrm{C}=\mathrm{O})$.

\section{5-Propargyloxy-1,3,4-triphenyl-2-pyrazole (2b)}

(35\%), yellowish oil. ${ }^{1} \mathrm{H}$ NMR (400 $\left.\mathrm{MHz}^{\mathrm{CDCl}}{ }_{3}\right) \delta 7.91$ $(2 \mathrm{H}, \mathrm{d}, J=7.8 \mathrm{~Hz}, \mathrm{Ar}-\mathrm{H}), 7.58-7.30(13 \mathrm{H}, \mathrm{m}, \mathrm{Ar}-\mathrm{H})$, $4.40\left(2 \mathrm{H}, \mathrm{d}, J=2.3 \mathrm{~Hz}, \mathrm{OCH}_{2} \mathrm{C} \equiv \mathrm{CH}\right), 2.36(1 \mathrm{H}, \mathrm{t}, J=2.3$ $\left.\mathrm{Hz}, \quad \mathrm{OCH}_{2} \mathrm{C} \equiv \mathrm{CH}\right) .{ }^{13} \mathrm{C}$ NMR $\left(100.62 \mathrm{MHz} \quad \mathrm{CDCl}_{3}\right)$ $\delta 149.5[\mathrm{~N}=C(\mathrm{Ph}) \mathrm{C}(\mathrm{Ph})=\mathrm{C}], 149.0[\mathrm{~N}=\mathrm{C}(\mathrm{Ph}) \mathrm{C}(\mathrm{Ph})=$ C], 138.5 (Ar), 133.3 (Ar), 131.6 (Ar), 129.9 (Ar), 128.9 (Ar), 128.5 (Ar), 128.1 (Ar), 128.0 (Ar), 127.7 (Ar), 127.1 (Ar), $126.8(\mathrm{Ar}), 122.8(\mathrm{Ar}), 107.9[\mathrm{~N}=\mathrm{C}(\mathrm{Ph}) C(\mathrm{Ph})=\mathrm{C}]$, $77.1\left(\mathrm{OCH}_{2} \mathrm{C} \equiv \mathrm{CH}\right), 76.8\left(\mathrm{OCH}_{2} \mathrm{C} \equiv \mathrm{CH}\right), 61.0\left(\mathrm{OCH}_{2}\right.$ $\mathrm{C} \equiv \mathrm{CH}) . \mathrm{IR}$ (film, $\mathrm{cm}^{-1}$ ): 3291, 2125.

\section{General procedure for the thermal rearrangements of pyrazolinones and isomers}

In a round bottom flask, the $N$-substituted pyrazolinone or its $\mathrm{O}$-substituted isomer dissolved in $\mathrm{o}$-dichlorobenzene (ca. $0.04-0.05 \mathrm{M}$ ), are heated at $180^{\circ} \mathrm{C}$ until total consumption of the starting material (TLC control). After solvent evaporation the products are purified by flash chromatography $\left[\mathrm{Et}_{2} \mathrm{O} / n\right.$-hexane (1:4)].

\section{Pyrazolinone $4 a$ from $1 a$}

Following the general procedure, heating 1a (30 mg) for $60 \mathrm{~min}$ afforded 3,4-diphenyl-1-methyl-4-(prop-1',2' dienyl) pyrazolin-5-one (4a) (10.5 $\mathrm{mg}, 35 \%)$ as a colourless crystals: mp $146-147^{\circ} \mathrm{C}\left(\mathrm{Et}_{2} \mathrm{O}\right) .{ }^{1} \mathrm{H}$ NMR (400 $\left.\mathrm{MHz} \mathrm{CDCl}_{3}\right) \delta 7.56(2 \mathrm{H}, \mathrm{d}, J=7.0 \mathrm{~Hz}, \mathrm{Ar}-\mathrm{H}), 7.37-$ $7.26(8 \mathrm{H}, \mathrm{m}, \mathrm{Ar}-\mathrm{H}), 5.79(1 \mathrm{H}, \mathrm{t}, J=6.6 \mathrm{~Hz},(\mathrm{CH}=\mathrm{C}=$ $\left.\mathrm{CH}_{2}\right), 4.90\left(1 \mathrm{H}, \mathrm{dd}, J=11.6,6.6 \mathrm{~Hz}, \mathrm{CH}=\mathrm{C}=\mathrm{CH}_{2}\right), 4.74$ $\left(1 \mathrm{H}, \mathrm{dd}, J=11.6,6.6 \mathrm{~Hz}, \mathrm{CH}=\mathrm{C}=\mathrm{CH}_{2}\right), 3.45(3 \mathrm{H}, \mathrm{s}$, $\left.\mathrm{NCH}_{3}\right) .{ }^{13} \mathrm{C} \mathrm{NMR}\left(100.62 \mathrm{MHz} \mathrm{CDCl}_{3}\right) \delta 208.9,174.5$, 158.8, 136.1, 129.9, 129.2, 128.4, 128.2, 127.0, 89.1, 79.1, 60.1, 31.8. IR (film, $\mathrm{cm}^{-1}$ ) : 3061, 2921, $1956(\mathrm{C}=\mathrm{C}=\mathrm{C}$ ), 1714 $(\mathrm{C}=\mathrm{O})$, 1496.EIMS (m/z, \%): $288\left(\mathrm{M}^{+}, 60\right), 230$ (40), 178 (20), 128 (65), 77 (100). HRMS: calcd for $\mathrm{C}_{19} \mathrm{H}_{16} \mathrm{~N}_{2} \mathrm{O}$ : 288.126263; found: 288.126455. 


\section{Pyrazolinone $4 b$ from $1 b$}

Following the general procedure, heating $\mathbf{1 b}(14 \mathrm{mg})$ for 10 min afforded 4-(prop-1',2'-dienyl)-1,3,4-triphenylpyrazolin5-one (4b) (10.1 mg, 72\%) as a colourless oil. ${ }^{1} \mathrm{H}$ NMR (400 $\left.\mathrm{MHz} \mathrm{CDCl}_{3}\right) \delta 8.07(2 \mathrm{H}, \mathrm{d}, J=7.8 \mathrm{~Hz}, \mathrm{Ar}-\mathrm{H}), 7.70(2 \mathrm{H}, \mathrm{d}$, $J=7.8 \mathrm{~Hz}, \mathrm{Ar}-\mathrm{H}), 7.47-7.23(11 \mathrm{H}, \mathrm{m}, \mathrm{Ar}-\mathrm{H}), 5.90(1 \mathrm{H}, \mathrm{t}$, $\left.J=6.7 \mathrm{~Hz}, \mathrm{CH}=\mathrm{C}=\mathrm{CH}_{2}\right), 4.94(1 \mathrm{H}, \mathrm{dd}, J=11.7,6.7 \mathrm{~Hz}$, $\left.\mathrm{CH}=\mathrm{C}=\mathrm{CH}_{2}\right), 4.77(1 \mathrm{H}, \mathrm{dd}, J=11.7,6.7 \mathrm{~Hz}, \mathrm{CH}=\mathrm{C}=$ $\left.\mathrm{CH}_{2}\right) .{ }^{13} \mathrm{C}$ NMR $(100.62 \mathrm{MHz} \mathrm{CDCl} 3) \delta 209.0(\mathrm{CH}=\mathrm{C}=$ $\left.\mathrm{CH}_{2}\right), 172.7(\mathrm{C}=\mathrm{O}), 159.1(\mathrm{~N}=\mathrm{C}), 138.2,136.0,130.2$, 130.1, 129.3, 128.9, 128.4, 127.4, 127.0, 125.3, 119.1, 89.1 $\left(\mathrm{CH}=\mathrm{C}=\mathrm{CH}_{2}\right), 79.5\left(\mathrm{CH}=\mathrm{C}=\mathrm{CH}_{2}\right), 61.6(\mathrm{C}-\mathrm{CH}=\mathrm{C}=$ $\mathrm{CH}_{2}$ ). IR (film, $\left.\mathrm{cm}^{-1}\right): 3063,2921,1954(\mathrm{C}=\mathrm{C}=\mathrm{C}), 1721$ $(\mathrm{C}=\mathrm{O}), 1596,1494$. EIMS $(\mathrm{m} / \mathrm{z}, \%): 350\left(\mathrm{M}^{+}, 25\right), 230$ (12), 178 (10), 128 (21), 91 (32), 77 (100). HRMS: calcd for $\mathrm{C}_{24} \mathrm{H}_{18} \mathrm{~N}_{2} \mathrm{O}$ : 350.141913; found: 350.142194 .

\section{Pyrazolinone $4 a$ from isomer $2 a$}

Following the general procedure, heating $\mathbf{2 a}(20 \mathrm{mg})$ for $10 \mathrm{~min}$ afforded 3,4-diphenyl-1-methyl-4-(prop-1',2'dienyl)pyrazolin-5-one (4a) (18 $\mathrm{mg}, 90 \%)$ as a white solid with data identical with 4.4.1.

\section{Pyrazolinone $4 b$ from isomer $2 b$}

Following the general procedure, heating $\mathbf{2 b}(24 \mathrm{mg})$ for 10 min afforded 4-(prop-1',2'-dienyl)-1,3,4-triphenylpyrazolin5-one (4b) (24 mg, 99\%) as a white solid with data identical with 4.4.2.

\section{Pyrazolinone $6 a$ from $5 a$}

Following the general procedure, heating $\mathbf{5 a}(10 \mathrm{mg})$ for $30 \mathrm{~min}$ afforded the pyrazolinone $\mathbf{6 a}(3 \mathrm{mg}, 30 \%)$ as a glassy solid. ${ }^{1} \mathrm{H}$ NMR $\left(400 \mathrm{MHz} \mathrm{CDCl}_{3}\right) \delta 7.52(2 \mathrm{H}, \mathrm{d}$, $J=7.3 \mathrm{~Hz}, \mathrm{Ar}-\mathrm{H}), 7.38-7.22(8 \mathrm{H}, \mathrm{m}, \mathrm{Ar}-\mathrm{H}), 3.47(3 \mathrm{H}$, s, $\left.\mathrm{NCH}_{3}\right), 3.43\left(1 \mathrm{H}, \mathrm{dd}, J=16.0 \mathrm{~Hz}, 2.5 \mathrm{~Hz}, \mathrm{CH}_{2} \mathrm{C} \equiv \mathrm{CH}\right)$, $3.05\left(1 \mathrm{H}, \mathrm{dd}, J=16.0,2.5 \mathrm{~Hz}, \mathrm{CH}_{2} \mathrm{C} \equiv \mathrm{CH}\right), 1.91(1 \mathrm{H}, \mathrm{t}$, $\left.J=2.5 \mathrm{~Hz}, \mathrm{CH}_{2} \mathrm{C} \equiv \mathrm{CH}\right)$. IR (film, $\left.\mathrm{cm}^{-1}\right): 3291(\mathrm{C} \equiv \mathrm{C}-\mathrm{H})$, 2923, $1956(\mathrm{C} \equiv \mathrm{C}), 1713(\mathrm{C}=\mathrm{O})$.

\section{Pyrazolinone $6 a$ from isomer $7 a$}

Following the general procedure, heating $7 \mathbf{a}(10 \mathrm{mg})$ for 10 min afforded $6 \mathbf{6}(8 \mathrm{mg}, 80 \%)$ with data identical with the previous reaction.

\section{Reactions of pyrazolinones with base}

In a round bottom flask, the propargylated pyrazolinone 1a or its isomer 2a is dissolved in dry THF (ca. $0.07 \mathrm{M}$ ) ( 1 eq.) under argon and 18-crown- 6 ether (1 eq.) is added. After total dissolution of the reactants, EtOK (0.1 eq.) is added under anhydrous conditions. The reaction is left to react at room temperature. After consumption of the starting material (TLC control) the reaction is stopped by addition of a saturated solution of $\mathrm{NH}_{4} \mathrm{Cl}$ and extraction with $\mathrm{Et}_{2} \mathrm{O}(2 \mathrm{x})$. The product is purified by flash chromatography [AcOEt / $n$-hexane (1:6)].

\section{Pyrazolinone $6 a$ from isomer $7 a$}

Following the general procedure, 3,4-diphenyl-1-methyl2-(prop-1',2'-dienyl)pyrazolin-5-one (5a) was obtained from 1a after $16 \mathrm{~h}$ as a colourless oil (65\%). ${ }^{1} \mathrm{H}$ NMR $\left(400 \mathrm{MHz} \mathrm{CDCl}_{3}\right) \delta 7.69-7.64(4 \mathrm{H}, \mathrm{m}, \mathrm{Ar}-\mathrm{H}), 7.56-$ $7.52(2 \mathrm{H}, \mathrm{m}, \mathrm{Ar}-\mathrm{H}), 7.47-7.43(4 \mathrm{H}, \mathrm{m}, \mathrm{Ar}-\mathrm{H}), 6.30$ $\left(1 \mathrm{H}, \mathrm{t}, J=6.2 \mathrm{~Hz}, \mathrm{~N}-\mathrm{CH}=\mathrm{C}=\mathrm{CH}_{2}\right), 5.32(2 \mathrm{H}, \mathrm{d}, J=6.2$ $\left.\mathrm{Hz}, \mathrm{NCH}=\mathrm{C}=\mathrm{CH}_{2}\right), 3.49\left(3 \mathrm{H}, \mathrm{s}, \mathrm{NCH}_{3}\right)$. IR (film, cm ${ }^{-1}$ ): 3055, 2925, $1962(\mathrm{C}=\mathrm{C}=\mathrm{C}), 1659(\mathrm{C}=\mathrm{O}), 1483$.

\section{Isomer $7 a$ from $2 a$}

Following the general procedure, 1-methyl-3,4-diphenyl5-(prop-1', 2' -dienyloxy)pyrazole (7a) was obtained from 2a after $16 \mathrm{~h}$ as a colourless oil (74\%). ${ }^{1} \mathrm{H}$ NMR (400 $\left.\mathrm{MHz} \mathrm{CDCl}_{3}\right) \delta 7.47-7.43(2 \mathrm{H}, \mathrm{m}, \mathrm{Ar}-\mathrm{H}), 7.34-7.24$ $(8 \mathrm{H}, \mathrm{m}, \mathrm{Ar}-\mathrm{H}), 6.79\left(1 \mathrm{H}, \mathrm{t}, J=5.9 \mathrm{~Hz}, \mathrm{OCH}=\mathrm{C}=\mathrm{CH}_{2}\right)$, $5.36\left(2 \mathrm{H}, \mathrm{d}, J=5.9 \mathrm{~Hz}, \mathrm{OCH}=\mathrm{C}=\mathrm{CH}_{2}\right), 3.81(3 \mathrm{H}, \mathrm{s}$, $\left.\mathrm{NCH}_{3}\right) .{ }^{13} \mathrm{C}$ NMR $\left(100.62 \mathrm{MHz} \mathrm{CDCl}_{3}\right) \delta 200.0(\mathrm{OCH}=$ $\left.C=\mathrm{CH}_{2}\right), 147.7,147.5,133.5$ (Ar), 131.6, 129.6, 129.2, 128.3, 128.2, 127.9, 127.6, 126.7, $121.7\left(\mathrm{OCH}=\mathrm{C}=\mathrm{CH}_{2}\right)$, 93.0 $\left(\mathrm{OCH}=\mathrm{C}=\mathrm{CH}_{2}\right), 34.7\left(\mathrm{NCH}_{3}\right)$. IR (film, $\left.\mathrm{cm}^{-1}\right)$ : 3059, 2940, $1972(\mathrm{C}=\mathrm{C}=\mathrm{C}), 1605,1563$.

\section{Synthesis of isoxazolinones}

\section{Diphenyl-2-(prop-2'-yn-1'-yl)isoxazolin-5-one (8a)}

To a solution of 3,4-diphenylisoxazolin-5-one (Section 4.2.4) (100 mg, $0.422 \mathrm{mmol}$ ), triphenylphosphine (121 mg, $0.46 \mathrm{mmol})$ and propargylic alcohol $(21.5 \mathrm{mg}, 0.384 \mathrm{mmol})$ in dry THF (1.5 mL) were added, dropwise, DIAD $(93 \mathrm{mg}$, $0.460 \mathrm{mmol}$ ). After the addition was completed, the solvent was removed under reduced pressure and the crude purified by $\mathrm{CC}$ (silica; $\mathrm{Et}_{2} \mathrm{O} / n$-hexane $1: 2$ ) to yield the title compound 8a $(22 \mathrm{mg}, 21 \%): \mathrm{mp} 81-82^{\circ} \mathrm{C}\left(\mathrm{Et}_{2} \mathrm{O} / \mathrm{n}\right.$ hexane). IR ( $\left.\mathrm{KBr}, \mathrm{cm}^{-1}\right): 3291$ ( $\left.\mathrm{HC} \equiv \mathrm{C}\right), 2127(\mathrm{C} \equiv \mathrm{C}), 1746$ $(\mathrm{C}=\mathrm{O}) .{ }^{1} \mathrm{H}$ NMR $\left(400 \mathrm{MHz} \mathrm{CDCl}_{3}\right) \delta 2.64(1 \mathrm{H}, \mathrm{s}$, $\mathrm{HC} \equiv \mathrm{C}), 5.06\left(2 \mathrm{H}, \mathrm{d}, J=2.1 \mathrm{~Hz}, \mathrm{NCH}_{2}\right), 7.26-7.56(10 \mathrm{H}$, $\mathrm{m}, \operatorname{Ar}-\mathrm{H})$. EIMS (m/z, \%): $275\left(\mathrm{M}^{+}, 23\right), 178$ (34), 105 (100), 89 (60), 77 (50). HRMS calcd for $\mathrm{C}_{18} \mathrm{H}_{13} \mathrm{NO}_{2}$ : 275.09463; found: 275.09405 .

\section{Diphenyl-2-(2'-methylbut-3'-yn-2'-yl)isoxazolin-5-one (8b)}

Following Nicholas protocol [24], to a stirred solution of 2-methylbut-3-yn-2-ol (25.2 $\mathrm{mg}, 0.3 \mathrm{mmol})$ in petroleum ether $(2 \mathrm{~mL})$, under nitrogen at $\mathrm{RT}$, was added $\mathrm{Co}_{2}(\mathrm{CO})_{8}$ (102.6 mg, $0.3 \mathrm{mmol}$.) and MS $4 \AA$, followed by boron trifluoride diethyl etherate $(42.5 \mathrm{mg}, 0.3 \mathrm{mmol})$. After 10 min 3,4-diphenylisoxazolin-5-one (Section 4.2.4) (23.7 $\mathrm{mg}, 0.1 \mathrm{mmol}$ ) was added and the reaction monitored by TLC (silica; $\mathrm{CH}_{2} \mathrm{Cl}_{2} / \mathrm{AcOEt}$ 4:1) until consumption of the starting isoxazolone. After $48 \mathrm{~h}$ water $(5 \mathrm{~mL})$ was added to the reaction mixture which was extracted with 
$\mathrm{Et}_{2} \mathrm{O}(2 \times 5 \mathrm{~mL})$ to yield the cobalt complex as a red oil, which was purified by PTLC (silica; $n$-hexane / AcOEt 4:1). To a solution of this red complex in acetone $(2 \mathrm{~mL})$ was added $\mathrm{Et}_{3} \mathrm{~N}(0.1 \mathrm{mmol})$ and $\left(\mathrm{NH}_{4}\right)_{2} \mathrm{Ce}\left(\mathrm{NO}_{3}\right)_{6}$ (55 mg, $0.1 \mathrm{mmol}$ ) until formation of the required isoxazolinone. Water was added $(10 \mathrm{~mL})$, followed by extraction of the mixture with $\mathrm{Et}_{2} \mathrm{O}(3 \times 5 \mathrm{~mL})$ and purification by PTLC (silica; $\mathrm{Et}_{2} \mathrm{O} / n$-hexane 1:1) afforded the $N$-substituted isoxazolinone $\mathbf{8 b}(17.6 \mathrm{mg}$, 58\%): $\mathrm{mp} 113-115^{\circ} \mathrm{C}\left(\mathrm{Et}_{2} \mathrm{O} / \mathrm{n}\right.$-hexane). IR $\left(\mathrm{KBr}, \mathrm{cm}^{-1}\right)$ : 3291 ( $\mathrm{HC} \equiv \mathrm{C}), 2113(\mathrm{C} \equiv \mathrm{C}), 1746$ (s, C=O). ${ }^{1} \mathrm{H}$ NMR $\left(400 \mathrm{MHz} \mathrm{CDCl}_{3}\right) \delta 1.48\left(6 \mathrm{H}, \mathrm{s}, \mathrm{CH}_{3}\right), 2.31(1 \mathrm{H}, \mathrm{s}$, $\mathrm{HC} \equiv \mathrm{C}), 7.19-7.50(10 \mathrm{H}, \mathrm{m}, \mathrm{Ar}-\mathrm{H}) .{ }^{13} \mathrm{C}-\mathrm{NMR}(100.62$ $\left.\mathrm{MHz} \mathrm{CDCl}_{3}\right) \delta 28.5\left[\mathrm{NC}\left(\mathrm{CH}_{3}\right)_{2}\right], 61.3\left[\mathrm{NC}\left(\mathrm{CH}_{3}\right)_{2}\right], 73.7$ $(\mathrm{HC} \equiv \mathrm{C}), 82.4(\mathrm{HC} \equiv \mathrm{C}), 127.4,128.2,128.3,128.7,129.5$, 130.6, 170.2. EIMS (m/z, \%): $303\left(\mathrm{M}^{+}, 17\right), 237$ $\left(\mathrm{C}_{15} \mathrm{H}_{11} \mathrm{NO}_{2}^{+}, 100\right), 178$ (35), 67 (40). HRMS: calcd for $\mathrm{C}_{20} \mathrm{H}_{17} \mathrm{NO}_{2}$ : 303.12593; found: 303.12641 .

\section{Allyl-3-methyl-4-phenylisoxazolin-5-one (8c)}

To 3-methyl-4-phenylisoxazolin-5-one (Section 4.2.3) $(0.3 \mathrm{~g}, 1.71 \mathrm{mmol})$ was added a solution of $\mathrm{Na}(39 \mathrm{mg})$ in EtOH $(1.7 \mathrm{~mL})$. To this solution was added allyl bromide $(0.207 \mathrm{mg}, 1.71 \mathrm{mmol})$. The reaction mixture was magnetically stirred, under argon, while being heated under reflux $(1.8 \mathrm{~h})$. On completion of the reaction (TLC control: silica; 1) $\mathrm{Et}_{2} \mathrm{O} / n$-hexane 1:1, 2) AcOEt), the solvent was removed under reduced pressure. Purification by $\mathrm{CC}$ (silica; $\mathrm{Et}_{2} \mathrm{O} / n$-hexane 1:1) afforded the title compound $\mathbf{8 c}(171,6 \mathrm{mg}, 66 \%)$ as a white solid: $\mathrm{mp} 50-51^{\circ} \mathrm{C}$ ( $\mathrm{Et}_{2} \mathrm{O} / n$-hexane). IR ( $\mathrm{KBr}$, $\mathrm{cm}^{-1}$ ): To 3-methyl-4-phenylisoxazolin-5-one (Section 4.2.3) (0.3 g, $1.71 \mathrm{mmol}$ ) was added a solution of $\mathrm{Na}$ (39 $\mathrm{mg})$ in EtOH $(1.7 \mathrm{~mL})$. To this solution was added allyl bromide $(0.207 \mathrm{mg}, 1.71 \mathrm{mmol})$. The reaction mixture was magnetically stirred, under argon, while being heated under reflux $(1.8 \mathrm{~h})$. On completion of the reaction (TLC control: silica; 1) $\mathrm{Et}_{2} \mathrm{O} / n$-hexane $1: 1,2$ ) AcOEt), the solvent was removed under reduced pressure. Purification by $\mathrm{CC}$ (silica; $\mathrm{Et}_{2} \mathrm{O} / n$-hexane 1:1) afforded the title compound 8c (171,6 $\mathrm{mg}, 66 \%)$ as a white solid: $\mathrm{mp} 50-51^{\circ} \mathrm{C}\left(\mathrm{Et}_{2} \mathrm{O} / n\right.$-hexane). IR ( $\mathrm{KBr}$, $\left.\mathrm{cm}^{-1}\right): 1711(\mathrm{C}=\mathrm{O}), 1604(\mathrm{C}=\mathrm{C}) .{ }^{1} \mathrm{H}$ NMR $(400 \mathrm{MHz}$ $\left.\mathrm{CDCl}_{3}\right) \delta 2.29\left(3 \mathrm{H}, \mathrm{s}, \mathrm{CH}_{3}\right), 4.27\left(2 \mathrm{H}, \mathrm{d}, \mathrm{J} 6 \mathrm{~Hz}, \mathrm{NCH}_{2}\right)$, $5.30\left(2 \mathrm{H}, \mathrm{m}, \mathrm{CH}_{2}=\mathrm{CH}\right), 5.80\left(1 \mathrm{H}, \mathrm{m}, \mathrm{CH}_{2}=\mathrm{CH}\right), 7.27$ $(1 \mathrm{H}, \mathrm{t}, J=7.4 \mathrm{~Hz}, p-\mathrm{Ar}-\mathrm{H}), 7.38(2 \mathrm{H}, \mathrm{t}, J=7.5 \mathrm{~Hz}$, $m-\mathrm{Ar}-\mathrm{H}), 7.46(2 \mathrm{H}, \mathrm{d}, J=7.6 \mathrm{~Hz}, o-\mathrm{Ar}-\mathrm{H}) .{ }^{13} \mathrm{C}-\mathrm{NMR}$ $\left(100.62 \mathrm{MHz} \mathrm{CDCl}_{3}\right) \delta 11.8\left(\mathrm{CH}_{3}\right), 53.1\left(\mathrm{~N}-\mathrm{CH}_{2}\right), 103.8$ $[\mathrm{N}-\mathrm{C}(\mathrm{Me})=\mathrm{C}(\mathrm{Ph})], 120.5\left(\mathrm{CH}_{2}=\mathrm{CH}\right), 127.2(\mathrm{Ar}), 128.2$ (Ar), 128.5 (Ar), 129.4 (Ar), $129.6\left(\mathrm{CH}_{2}=\mathrm{CH}\right), 159.3$ $[\mathrm{N}-\mathrm{C}(\mathrm{Me})=\mathrm{C}(\mathrm{Ph})], \quad 169.5 \quad(\mathrm{C}=\mathrm{O})$. EIMS $(\mathrm{m} / \mathrm{z}, \%)$ : 215 (M+100), 130 (21), 116 (38), 115 (46), 89 (13), 77 (13). HRMS: calcd for $\mathrm{C}_{13} \mathrm{H}_{13} \mathrm{NO}_{2}$ : 215.09463; found: 215.09397.

\section{Allyl-3,4-diphenylisoxazolin-5-one (8d)}

3,4-Diphenylisoxazolin-5-one (Section 4.2.4) (0.406 g, $1.71 \mathrm{mmol})$, using the previous protocol, afforded the title compound (8d) (256 mg, 85\%) as a white solid: $\mathrm{mp}$ $105-106^{\circ} \mathrm{C}\left(\mathrm{Et}_{2} \mathrm{O} / \mathrm{n}\right.$-hexane). IR $\left(\mathrm{KBr}, \mathrm{cm}^{-1}\right): 1720$ ( $\mathrm{C}=$ O), $1615(\mathrm{C}=\mathrm{C}) .{ }^{1} \mathrm{H}$ NMR $\left.\left(400 \mathrm{MHz}^{\mathrm{CDCl}}\right)_{3}\right) \delta 4.06$ $\left(2 \mathrm{H}, \mathrm{d}, J 6.0 \mathrm{~Hz}, \mathrm{NCH}_{2}\right), 5.16\left(1 \mathrm{H}, d, J=17.2 \mathrm{~Hz}, \mathrm{CH}_{2}=\right.$ $\mathrm{CH}), 5.25\left(1 \mathrm{H}, \mathrm{d}, J=10.8 \mathrm{~Hz}, \mathrm{CH}_{2}=\mathrm{CH}\right), 5.78(1 \mathrm{H}, \mathrm{m}$, $\left.\mathrm{CH}_{2}=\mathrm{CH}\right), 7.18-7.48(10 \mathrm{H}, \mathrm{m}, \mathrm{Ar}-\mathrm{H}) \cdot{ }^{13} \mathrm{C} \mathrm{NMR}$ $\left(100.62 \mathrm{MHz} \mathrm{CDCl}_{3}\right) \delta 54.9\left(\mathrm{~N}-\mathrm{CH}_{2}\right), 105.2[\mathrm{~N}-\mathrm{C}(\mathrm{Ph})=$ $C(\mathrm{Ph})], 121.0\left(\mathrm{CH}_{2}=\mathrm{CH}\right), 127.2(\mathrm{Ar}), 127.7(\mathrm{Ar}), 128.2$ (Ar), 128.3(Ar), 128.7(Ar), 129.0(Ar), 129.2(Ar), 129.3(Ar), $131.0\left(\mathrm{CH}_{2}=\mathrm{CH}\right), 162.8$ [N-C(Ph)], $169.9(\mathrm{C}=\mathrm{O})$. EIMS (m/z, \%): 277 (M $\left.\mathrm{M}^{+}, 100\right), 236$ (12), 192 (27), 178 (76), 133 (13), 117 (51), 89 (46), 77 (22). HRMS: calcd for $\mathrm{C}_{18} \mathrm{H}_{15} \mathrm{NO}_{2}$ : 277.11028; found: 277.11037.

\section{Synthesis of isoxazolidinone $8 e$}

Diethyl 2-[(allyl-(tert-butyldimethylsilyloxy)amino) methylene]malonate (10) To a stirred solution of $N$ tert-butyldimethylsilyloxyprop-2-en-1-amine (0.5 g, 2.67 mmol) in $\mathrm{CCl}_{4} / \mathrm{CHCl}_{3}(1: 3)(20 \mathrm{~mL})$ under argon was added diethyl 2-(ethoxymethylene)malonate [7] (0.58 g, $2.67 \mathrm{mmol}, 1$ eq.). On completion of the reaction (TLC control: silica, $\mathrm{Et}_{2} \mathrm{O} / \mathrm{n}$-hexane 1:1) (30 h), the solvent was removed under reduced pressure by $\mathrm{CC}$ (silica; $\mathrm{Et}_{2} \mathrm{O} /$ $n$-hexane 1:1) to afford the title compound $\mathbf{1 0}(50 \%)$ as an oil (50\%): IR (film, cm $\left.{ }^{-1}\right) 1732(\mathrm{C}=\mathrm{O}), 1707(\mathrm{C}=\mathrm{O}), 1621$ $(\mathrm{C}=\mathrm{C}), 1262(\mathrm{Si}-\mathrm{O}-\mathrm{N}) .{ }^{1} \mathrm{H}$ NMR $\left(400 \mathrm{MHz} \mathrm{CDCl}_{3}\right) \delta$ $0.19\left[6 \mathrm{H}, \mathrm{s}, \mathrm{Si}\left(\mathrm{CH}_{3}\right)_{2}\right], 0.93\left[9 \mathrm{H}, \mathrm{s}, \mathrm{C}\left(\mathrm{CH}_{3}\right)_{3}\right], 1.23(6 \mathrm{H}, \mathrm{m}$, $\left.\mathrm{OCH}_{2} \mathrm{CH}_{3}\right), 4.00\left(2 \mathrm{H}, \mathrm{d}, J=6.0 \mathrm{~Hz}, \mathrm{NCH}_{2}\right), 4.12(4 \mathrm{H}$, $\left.\mathrm{m}, \mathrm{OCH}_{2} \mathrm{CH}_{3}\right), 5.26\left(1 \mathrm{H}, \mathrm{d}, J=10.4 \mathrm{~Hz}, \mathrm{CH}_{2}=\mathrm{CH}\right), 5.35$ $\left(1 \mathrm{H}, \mathrm{d}, J=17.3 \mathrm{~Hz}, \mathrm{CH}_{2}=\mathrm{CH}\right), 5.90\left(1 \mathrm{H}, \mathrm{m}, \mathrm{CH}_{2}=\mathrm{CH}\right)$, $7.88(1 \mathrm{H}, \mathrm{s}, \mathrm{N}-\mathrm{CH}=\mathrm{C}) .{ }^{13} \mathrm{C} \mathrm{NMR}\left(100.62 \mathrm{MHz}^{\mathrm{CDCl}} \mathrm{CD}_{3}\right)$ $\delta$-5.3 $\left[\mathrm{Si}\left(\mathrm{CH}_{3}\right)_{2}\right], 14.1\left(\mathrm{OCH}_{2} \mathrm{CH}_{3}\right), 17.8\left[\mathrm{C}\left(\mathrm{CH}_{3}\right)_{3}\right], 25.8$ $\begin{array}{lllll}{\left[\mathrm{C}\left(\mathrm{CH}_{3}\right)_{3}\right.} & \end{array}, \quad 59.9 \quad\left(\mathrm{CH}_{2} \mathrm{~N}\right), \quad 60.3 \quad\left(\mathrm{OCH}_{2} \mathrm{CH}_{3}\right), \quad 60.6$ $\left(\mathrm{OCH}_{2} \mathrm{CH}_{3}\right), 61.4(\mathrm{NCH}=\mathrm{C}), 119.6\left(\mathrm{CH}_{2}=\mathrm{CH}\right), 131.1$ $\left(\mathrm{CH}_{2}=\mathrm{CH}\right), 149.5(\mathrm{~N}-\mathrm{CH}), 166.2(\mathrm{C}=\mathrm{O})$. EIMS (m/z, \%): $357\left(\mathrm{M}^{+}, 66\right), 312\left(\left[\mathrm{M}-\mathrm{C}_{2} \mathrm{H}_{5} \mathrm{O}\right]^{+}, 86\right), 300\left(\left[\mathrm{M}-\mathrm{C}_{4} \mathrm{H}_{9}\right]^{+}\right.$, 100), 226 ([M- $\left.\left.\mathrm{C}_{6} \mathrm{H}_{15} \mathrm{OSi}\right]^{+}, 97\right), 198(20), 57\left(\mathrm{C}_{4} \mathrm{H}_{9}^{+}, 49\right)$. HRMS: calcd for $\mathrm{C}_{17} \mathrm{H}_{31} \mathrm{NO}_{5} \mathrm{Si}$ : 357.19715; found: 357.19796 .

Diethyl 2-[(allyl-(tert-butyldimethylsilyloxy)amino) methylene]malonate (10) To a magnetically stirred solution of 2-[(allyl-(tert-butyldimethylsilyloxy)amino) methylene] malonate [5] (10) (50 mg, $0.140 \mathrm{mmol})$ in $\mathrm{CH}_{3} \mathrm{CN}$ [6 $\mathrm{mL}, 0.1 \% \mathrm{H}_{2} \mathrm{O}(\mathrm{m} / \mathrm{v})$ ], at $\mathrm{RT}$ under nitrogen, was added $\mathrm{SbCl}_{5}\left(359 \mu \mathrm{L}\right.$, from $0.039 \mathrm{M}$ solution in $\mathrm{CH}_{3} \mathrm{CN}$, 0.1 eq.). On completion of the reaction $(10 \mathrm{~m})$, (TLC control: silica; $\mathrm{Et}_{2} \mathrm{O} / \mathrm{n}$-hexane $1: 1$ ), the solvent was removed under reduced pressure. Purification by $\mathrm{CC}$ (silica; $\mathrm{Et}_{2} \mathrm{O} / \mathrm{n}$-hexane 1:1) afforded diethyl 2-[(allyl 
(hydroxyl)amino) methylene]malonate (11) (23 mg, $68 \%)$, and ethyl 2-allyl-5-oxo-2,5-dihydroisoxazole-4carboxylate (8e) (3.3 mg, 12\%). Compound 11 was unstable and lactonised spontaneously on standing to the 2-allyl-isoxazolinone $\mathbf{8 e}$.

Ethyl 2-allyl-5-oxo-2,5-dihydroisoxazole-4-carboxylate (8e) Colourless crystals, mp $45-46^{\circ} \mathrm{C}$ (n-hexane). IR (film, $\left.\mathrm{cm}^{-1}\right)$ : $1784(\mathrm{C}=\mathrm{O}$, ester), $1698(\mathrm{C}=\mathrm{O}), 1578(\mathrm{C}$ =C). ${ }^{1} \mathrm{H}$ NMR $\left(400 \mathrm{MHz} \mathrm{CDCl}_{3}\right) \delta 1.30(3 \mathrm{H}, \mathrm{t}, J=7.1$ $\left.\mathrm{Hz}, \mathrm{OCH}_{2} \mathrm{CH}_{3}\right), 4.26\left(2 \mathrm{H}, \mathrm{q}, J=7.1 \mathrm{~Hz}, \mathrm{OCH}_{2} \mathrm{CH}_{3}\right), 4.47$ $\left(2 \mathrm{H}, \mathrm{d}, J=6.3 \mathrm{~Hz}, \mathrm{NCH}_{2}\right), 5.46\left(2 \mathrm{H}, \mathrm{m}, \mathrm{CH}_{2}=\mathrm{CH}\right), 5.89$ $\left(1 \mathrm{H}, \mathrm{m}, \mathrm{CH}_{2}=\mathrm{CH}\right), 8.34(\mathrm{H}, \mathrm{s}, \mathrm{N}-\mathrm{CH})$. Elemental analysis: calcd for $\mathrm{C}_{9} \mathrm{H}_{11} \mathrm{NO}_{4}$ (\%): C: 54.80, H: 5.63, N: 7.11 . Found (\%): C: 54.53, H: 5.66, N: 7.01.

Diethyl 2-[(N-allyl(hydroxyl)amino)methylene] malonate (11) Oil, IR (film, cm ${ }^{-1}$ ): 3600 - 1800 (l, OH), $1685(\mathrm{C}=\mathrm{O}), 1629(\mathrm{C}=\mathrm{C}) .{ }^{1} \mathrm{H}$ NMR $\left(400 \mathrm{MHz} \mathrm{CD}_{3} \mathrm{CN}\right)$ $\delta 1.26\left(3 \mathrm{H}, \mathrm{t}, J=7.1 \mathrm{~Hz}, \mathrm{OCH}_{2} \mathrm{CH}_{3}\right), 1.33(3 \mathrm{H}, \mathrm{t}, J=7.1$ $\left.\mathrm{Hz}, \mathrm{OCH}_{2} \mathrm{CH}_{3}\right), 4.16\left(2 \mathrm{H}, \mathrm{q}, J=7.1 \mathrm{~Hz}, \mathrm{OCH}_{2} \mathrm{CH}_{3}\right)$, $4.25\left(4 \mathrm{H}, \mathrm{m}, \mathrm{OCH}_{2} \mathrm{CH}_{3}+\mathrm{NCH}_{2}\right), 5.39\left(2 \mathrm{H}, \mathrm{m}, \mathrm{CH}_{2}=\right.$ $\mathrm{CH}), 5.96\left(1 \mathrm{H}, \mathrm{m}, \mathrm{CH}_{2}=\mathrm{CH}\right), 7.86(1 \mathrm{H}, \mathrm{s}, \mathrm{N}-\mathrm{CH}=\mathrm{C})$, $13.11\left(1 \mathrm{H}, \mathrm{s}, \mathrm{OH}\right.$, exchange $\left.\mathrm{D}_{2} \mathrm{O}\right) .{ }^{13} \mathrm{C}$ NMR $(100.62$ $\left.\mathrm{MHz} \quad \mathrm{CD}_{3} \mathrm{CN}\right) \quad \delta \quad 14.1 \quad\left(\mathrm{OCH}_{2} \mathrm{CH}_{3}\right), 30.2 \quad\left(\mathrm{CCO}_{2} \mathrm{Et}\right)$, $60.0\left(\mathrm{OCH}_{2} \mathrm{CH}_{3}\right), 61.5\left(\mathrm{OCH}_{2} \mathrm{CH}_{3}\right), 61.6\left(\mathrm{NCH}_{2}\right), 120.8$ $\left(\mathrm{CH}=\mathrm{CH}_{2}\right), 129.9\left(\mathrm{CH}=\mathrm{CH}_{2}\right), 147.3(\mathrm{NCH}=\mathrm{C}), 166.2$ $(\mathrm{C}=\mathrm{O})$. EIMS (m/z, \%) $243\left(\mathrm{M}^{+}, 2\right), 197\left(\mathrm{C}_{9} \mathrm{H}_{11} \mathrm{NO}_{4}^{+}\right.$, 100). HRMS: calcd for $\mathrm{C}_{11} \mathrm{H}_{17} \mathrm{NO}_{5}$ : 243.11067; found: 243.10985 .

\section{General procedure for the thermal rearrangements of isoxazolinones}

In a round bottom flask, the isoxazolinones (8a-e) dissolved in $o$-dichlorobenzene (ca. $0.04-0.05 \mathrm{M}$ ) are heated until total consumption of the starting material (TLC control). After solvent evaporation the products are purified by flash chromatography.

\section{Rearrangement of the 3,4-diphenyl-2-(prop-2'-yn-1'-yl) isoxazolin-5-one (8a)}

Following the general procedure, 3,4-diphenyl-4-(prop$1^{\prime}, 2^{\prime}$-dien-1-yl)isoxazolin-5-one (9a) was obtained by heating $8 \mathbf{a}(10 \mathrm{mg})$ at $60^{\circ} \mathrm{C}$ for $30 \mathrm{~m}$, followed by $\mathrm{CC}$ (silica; $\mathrm{Et}_{2} \mathrm{O} / n$-hexane $1: 1$ ), as an oil (75\%): IR (film, $\left.\mathrm{cm}^{-1}\right): 1965(\mathrm{C}=\mathrm{C}=\mathrm{C}), 1798(\mathrm{C}=\mathrm{O}) .{ }^{1} \mathrm{H}$ NMR (400 $\left.\mathrm{MHz} \mathrm{CDCl}_{3}\right) \delta 4.88(1 \mathrm{H}, \mathrm{dd}, J=6.6,12.1 \mathrm{~Hz}, \mathrm{CH}=\mathrm{C}=$ $\left.\mathrm{CH}_{2}\right), 5.04\left(1 \mathrm{H}, \mathrm{dd}, J=6.6,12.1 \mathrm{~Hz}, \mathrm{CH}=\mathrm{C}=\mathrm{CH}_{2}\right), 5.80$ $\left(1 \mathrm{H}, \mathrm{t}, J=6.6 \mathrm{~Hz}, \mathrm{HC}=\mathrm{C}=\mathrm{CH}_{2}\right), 7.30-7.58(10 \mathrm{H}, \mathrm{m}$, $\mathrm{Ar}-\mathrm{H}) .{ }^{13} \mathrm{C} \mathrm{NMR}\left(100.62 \mathrm{MHz} \mathrm{CDCl}_{3}\right) \delta 57.8[\mathrm{~N}=\mathrm{C}$ $(\mathrm{Ph}) \mathrm{C}], 80.6\left(\mathrm{CH}=\mathrm{C}=\mathrm{CH}_{2}\right), 88.0\left(\mathrm{CH}=\mathrm{C}=\mathrm{CH}_{2}\right), 126.9$ (Ar), 127.1 (Ar), 127.7 (Ar), 128.8 (Ar), 129.2 (Ar), 129.6 (Ar), $131.6(\mathrm{Ar}), 134.3(\mathrm{Ar}), 166.6(\mathrm{~N}=\mathrm{C}), 175.1(\mathrm{C}=\mathrm{O})$, $203.0\left[\mathrm{CH}=\mathrm{C}=\mathrm{C}\left(\mathrm{CH}_{3}\right)_{2}\right]$. EIMS (m/z, \%): $275\left(\mathrm{M}^{+}, 19\right)$,
231 ([M-CO $\left.]_{2}^{+}, 100\right), 172$ (89), 128 (99), 102 (89), 77 (82). HRMS: calcd for $\mathrm{C}_{18} \mathrm{H}_{13} \mathrm{NO}_{2}$ : 275.09463; found: 275.09556 .

\section{Rearrangement of 3,4-diphenyl-2-(2'-methylbut-3'-yn-2'-yl) isoxazolin-5-one (8b)}

Following the general procedure, 3,4-diphenyl-4-(3'methylbut-1',2'-dien-1'-yl)isoxazolin-5-one (9b) was obtained by heating $8 \mathrm{~b}$ at $60^{\circ} \mathrm{C}$ for $10 \mathrm{~m}$, followed by $\mathrm{CC}$ (silica; $\mathrm{Et}_{2} \mathrm{O} / \mathrm{n}$-hexane 1:1), as a colourless solid (85\%): $\mathrm{mp} 77-80\left(\mathrm{Et}_{2} \mathrm{O} / \mathrm{n}\right.$-hexane). IR $\left(\mathrm{KBr}, \mathrm{cm}^{-1}\right)$ : $1969(\mathrm{C}=\mathrm{C}=\mathrm{C}), 1790(\mathrm{C}=\mathrm{O}) .{ }^{1} \mathrm{H}$ NMR $(400 \mathrm{MHz}$ $\left.\mathrm{CDCl}_{3}\right) \delta 1.37\left(3 \mathrm{H}, \mathrm{s}, \mathrm{CH}_{3}\right), 1.62\left(3 \mathrm{H}, \mathrm{s}, \mathrm{CH}_{3}\right), 5.65(1 \mathrm{H}$, $\mathrm{t}, J=2.6 \mathrm{~Hz}, \mathrm{HC}=\mathrm{C}=\mathrm{C}), 7.28-7.54(10 \mathrm{H}, \mathrm{m}, \mathrm{Ar}-\mathrm{H})$. ${ }^{13} \mathrm{C}$ NMR (100.62 $\left.\mathrm{MHz} \mathrm{CDCl}_{3}\right) \quad \delta \quad 19.3\left(\mathrm{CH}_{3}\right), 19.7$ $\left(\mathrm{CH}_{3}\right), \quad 58.5[\mathrm{~N}=\mathrm{C}(\mathrm{Ph}) \mathrm{C}], \quad 86.6 \quad\left[\mathrm{CH}=\mathrm{C}=\mathrm{C}\left(\mathrm{CH}_{3}\right)_{2}\right]$, $102.2\left(\mathrm{CH}=\mathrm{C}=\mathrm{C}\left(\mathrm{CH}_{3}\right)_{2}\right), 127.0(\mathrm{Ar}), 127.7(\mathrm{Ar}), 128.1$ (Ar), 128.5 (Ar), 128.9 (Ar), 129.4 (Ar), 129.7 (Ar), 131.2 (Ar), 132.2 (Ar), 134.7 (Ar), $167.2(\mathrm{~N}=\mathrm{C}), 177.5(\mathrm{C}=\mathrm{O})$, $203.5\left(\mathrm{CH}=\mathrm{C}=\mathrm{C}\left(\mathrm{CH}_{3}\right)_{2}\right)$. EIMS $(\mathrm{m} / \mathrm{z}, \%) 303\left(\mathrm{M}^{+}, 1\right)$, $288\left(\left[\mathrm{M}-\mathrm{CH}_{3}\right]^{+}, 29\right), 259\left(\left[\mathrm{M}-\mathrm{CO}_{2}\right]^{+}, 53\right), 244\left(\mathrm{C}_{18} \mathrm{H}_{14} \mathrm{~N}^{+}\right.$, 50), $200\left(\mathrm{C}_{13} \mathrm{H}_{12} \mathrm{O}_{2}^{+}, 100\right), 141\left(\mathrm{C}_{11} \mathrm{H}_{9}^{+}, 71\right), 89\left(\mathrm{C}_{7} \mathrm{H}_{5}^{+}, 20\right)$, $77\left(\mathrm{C}_{6} \mathrm{H}_{5}^{+}, 76\right)$.

\section{Rearrangement of 2-allyl-3-methyl-4-phenylisoxazolin-5-one} (8c)

Following the general procedure, 4-allyl-3-methyl-4phenylisoxazolin-5-one (9c) was obtained by heating 8c at $180^{\circ} \mathrm{C}$ for $7 \mathrm{~h}$, followed by CC (silica; $\mathrm{Et}_{2} \mathrm{O} / \mathrm{n}$-hexane 1:1), as a viscous oil (95\%): IR (film, $\left.\mathrm{cm}^{-1}\right): 1791(\mathrm{C}=\mathrm{O})$, $1643(\mathrm{C}=\mathrm{N}) .{ }^{1} \mathrm{H}$ NMR $\left(400 \mathrm{MHz} \mathrm{CDCl}_{3}\right) \delta 1.95(3 \mathrm{H}, \mathrm{s}$, $\left.\mathrm{CH}_{3}\right), 2.85\left(1 \mathrm{H}, \mathrm{dd}, J=7.8,13.7 \mathrm{~Hz}, \mathrm{C}-\mathrm{CH}_{2}\right), 3.10(1 \mathrm{H}$, $\mathrm{dd}, J$ 6.7, $\left.13.7 \mathrm{~Hz}, \mathrm{C}-\mathrm{CH}_{2}\right), 5.25(1 \mathrm{H}, \mathrm{d}, J=10.1 \mathrm{~Hz}$, $\left.\mathrm{CH}_{2}=\mathrm{CH}\right), 5.30\left(1 \mathrm{H}, \mathrm{d}, J=16.9 \mathrm{~Hz}, \mathrm{CH}_{2}=\mathrm{CH}\right), 5.60$ $\left(1 \mathrm{H}, \mathrm{m}, \mathrm{CH}_{2}=\mathrm{CH}\right), 7.20-7.44(5 \mathrm{H}, \mathrm{m}, \mathrm{Ar}-\mathrm{H}) \cdot{ }^{13} \mathrm{C}$ NMR (100.62 MHz CDCl $) \delta 12.4\left(\mathrm{CH}_{3}\right), 36.4\left(\mathrm{C}-\mathrm{CH}_{2}\right)$, $59.3\left(\mathrm{C}-\mathrm{CH}_{2}\right), 121.4\left(\mathrm{CH}_{2}=\mathrm{CH}\right), 126.2(\mathrm{Ar})$, 127.3, 127.8, 128.9, 129.5, 129.7, $133.3\left(\mathrm{CH}_{2}=\mathrm{CH}\right), 168.3(\mathrm{C}=$ $\mathrm{N}), 178.7(\mathrm{C}=\mathrm{O})$. EIMS (m/z, \%): $215\left(\mathrm{M}^{+}, 100\right), 116$ (15), 115 (40), 77 (30). HRMS: calcd for $\mathrm{C}_{13} \mathrm{H}_{13} \mathrm{NO}_{2}$ : 215.09463; found: 215.09414.

\section{Rearrangement of 2-allyl-3,4-diphenylisoxazolin-5-one (8d)}

Following the general procedure, 4-allyl-3-methyl-3,4phenylisoxazolin-5-one (9d) was obtained by heating $\mathbf{8 d}$ at $180^{\circ} \mathrm{C}$ for $5 \mathrm{~h}$, followed by CC (silica; $\mathrm{Et}_{2} \mathrm{O} / n$-hexane $1: 1$ ), as a pale yellow solid (97\%): $\mathrm{mp} 71-72^{\circ} \mathrm{C}$. IR (KBr, $\left.\mathrm{cm}^{-1}\right): 1800$ (f, $\left.\mathrm{C}=\mathrm{O}\right), 1644(\mathrm{C}=\mathrm{N}) .{ }^{1} \mathrm{H}$ NMR $(400 \mathrm{MHz}$ $\left.\mathrm{CDCl}_{3}\right) \delta 3.03\left(1 \mathrm{H}, \mathrm{dd}, J=7.2,13.2 \mathrm{~Hz}, \mathrm{C}-\mathrm{CH}_{2}\right), 3.32$ $\left(1 \mathrm{H}, \mathrm{dd}, J=7.6,13.2 \mathrm{~Hz}, \mathrm{C}-\mathrm{CH}_{2}\right), 5.25(1 \mathrm{H}, \mathrm{d}, J=16,9$ $\left.\mathrm{Hz}, \mathrm{CH}_{2}=\mathrm{CH}\right), 5.14\left(1 \mathrm{H}, \mathrm{d}, J=10.1 \mathrm{~Hz}, \mathrm{CH}_{2}=\mathrm{CH}\right), 5.54$ $\left(1 \mathrm{H}, \mathrm{m}, \mathrm{CH}_{2}=\mathrm{CH}\right), 7.33-7.52(10 \mathrm{H}, \mathrm{m}, \mathrm{Ar}-\mathrm{H}) \cdot{ }^{13} \mathrm{C}$ NMR (100.62 $\left.\mathrm{MHz} \mathrm{CDCl}_{3}\right) \delta 37.5\left(\mathrm{C}-\mathrm{CH}_{2}\right), 58.8(\mathrm{C}-$ $\left.\mathrm{CH}_{2}\right), 121.8\left(\mathrm{CH}_{2}=\mathrm{CH}\right), 126.3(\mathrm{Ar}), 127.2(\mathrm{Ar}), 127.4$ 
(Ar), 128.9 (Ar), 129.0 (Ar), 129.4 (Ar), 129.7 (Ar), 131.7 $\left(\mathrm{CH}_{2}=\mathrm{CH}\right), 134.4(\mathrm{Ar}), 167.1(\mathrm{~N}=\mathrm{C}), 169.9(\mathrm{C}=\mathrm{O})$. EIMS (m/z, \%): $277\left(\mathrm{M}^{+}, 100\right), 236\left(\left[\mathrm{M}-\mathrm{C}_{3} \mathrm{H}_{5}\right]^{+}, 12\right), 232$ (21), 178 (49), 117 (51), 103 (35), 77 (30). HRMS: calcd for $\mathrm{C}_{18} \mathrm{H}_{15} \mathrm{NO}_{2}$ : 277.11028; found: 277.10956.

\section{Rearrangement of the ethyl 2-allyl-5-oxo-2,5- dihydroisoxazole-4-carboxylate (8e)}

Following the general procedure, ethyl 2-cyanopent-4enoate (12) was obtained by heating $8 \mathrm{e}$ at $180^{\circ} \mathrm{C}$ for $5 \mathrm{~h}$, followed by CC (silica; $\mathrm{Et}_{2} \mathrm{O} / n$-hexane 1:1), as a colourless oil [13] (80\%): IR (film, $\left.\mathrm{cm}^{-1}\right)$ : $2266(-\mathrm{C} \equiv \mathrm{N})$, $1750(\mathrm{C}=\mathrm{O}) .{ }^{1} \mathrm{H}$ NMR $\left(400 \mathrm{MHz} \mathrm{CDCl}_{3}\right) \delta 1.32(3 \mathrm{H}, \mathrm{t}$, $\left.J=7.1 \mathrm{~Hz}, \mathrm{OCH}_{2} \mathrm{CH}_{3}\right), 2.69\left(2 \mathrm{H}, \mathrm{m}, \mathrm{CHCH}_{2} \mathrm{CH}\right), 3.56(1 \mathrm{H}$, $\mathrm{t}, J=6.8 \mathrm{~Hz}, \mathrm{NC}-\mathrm{CH}), 4.27\left(2 \mathrm{H}, \mathrm{q}, J=7.1 \mathrm{~Hz}, \mathrm{OCH}_{2} \mathrm{CH}_{3}\right)$, $5.25\left(2 \mathrm{H}, \mathrm{m}, \mathrm{CH}_{2}=\mathrm{CH}\right), 5.83\left(1 \mathrm{H}, \mathrm{m}, \mathrm{CH}_{2}=\mathrm{CH}\right)$.

\section{Reaction of isoxazolinones with base}

Reaction of 2-allyl-3,4-diphenylisoxazolin-5-one (8d)

To a stirred solution of compound $8 \mathbf{8 d}(80 \mathrm{mg}, 0.289$ $\mathrm{mmol})$ in dry 1,4-dioxane $(2 \mathrm{~mL})$ at RT under argon, potassium ethoxide (1.5 eq., $36.5 \mathrm{mg}, 0.434 \mathrm{mmol}$ ) and 18crown-6 ether $(114.7 \mathrm{mg}, 0.434 \mathrm{mmol})$ were added. After $10 \mathrm{~m}$ the reaction was found complete (TLC control: silica; $\mathrm{Et}_{2} \mathrm{O} / n$-hexane 3:1), the mixture was neutralized with aq. $0.5 \mathrm{~N} \mathrm{AcOH}$ and the solvent removed under reduced pressure. The crude was diluted with $\mathrm{Et}_{2} \mathrm{O}(5 \mathrm{~mL})$ and washed with distilled water $(2 \mathrm{~mL})$. The organic layer was dried over anhydrous sodium sulphate, filtered and concentrated under reduced pressure. Purification by $\mathrm{CC}$ (silica; $\mathrm{Et}_{2} \mathrm{O} / \mathrm{n}$-hexane $\left.3: 1\right)$ afforded 2-(2'ethoxyethyl)-4,5-diphenyl-2,3-dihydro-1,3-oxazin -6-one (14) (23.3 mg, 25\%) as a yellow oil: IR (film, $\left.\mathrm{cm}^{-1}\right): 3257$ $(\mathrm{NH}), 1694(\mathrm{C}=\mathrm{O}), 1600,1585,1553 .{ }^{1} \mathrm{H}$ NMR $(400$ $\left.\mathrm{MHz} \mathrm{CDCl}_{3}\right) \delta 1.05\left(3 \mathrm{H}, \mathrm{t}, \mathrm{J}=7.0 \mathrm{~Hz}, \mathrm{OCH}_{2} \mathrm{CH}_{3}\right), 2.22$ $\left(2 \mathrm{H}, \mathrm{m}, \mathrm{CHCH}_{2}\right), 3.44\left(2 \mathrm{H}, \mathrm{m}, \mathrm{OCH}_{2} \mathrm{CH}_{3}\right), 3.62(1 \mathrm{H}, \mathrm{m}$, $\left.\mathrm{OCH}_{2} \mathrm{CH}_{2}\right), 3.77\left(1 \mathrm{H}, \mathrm{m}, \mathrm{OCH}_{2} \mathrm{CH}_{2}\right), 5.47(1 \mathrm{H}, \mathrm{q}, J=4.9$ $\left.\mathrm{Hz}, \mathrm{CHCH}_{2}\right), 6.46(1 \mathrm{H}, \mathrm{d}, J=4.1 \mathrm{~Hz}, \mathrm{NH}-$ exchange $\left.\mathrm{D}_{2} \mathrm{O}\right), 7.02-7.24(10 \mathrm{H}, \mathrm{m}, \mathrm{Ar}-\mathrm{H}) .{ }^{13} \mathrm{C}$ NMR $(100.62$ $\left.\mathrm{MHz} \mathrm{CDCl}_{3}\right) \delta 15.0\left(\mathrm{OCH}_{2} \mathrm{CH}_{3}\right), 32.0\left(\mathrm{OCH}_{2} \mathrm{CH}_{2}\right), 65.7$ $\left(\mathrm{OCH}_{2} \mathrm{CH}_{3}\right), 66.6\left(\mathrm{OCH}_{2} \mathrm{CH}_{2}\right), 81.6(\mathrm{~N}-\mathrm{CH}-\mathrm{O}), 103.9$ $(\mathrm{N}-\mathrm{C}=\mathrm{C}-\mathrm{CO}), 126.1(\mathrm{Ar}), 127.6(\mathrm{Ar}), 128.2(\mathrm{Ar}), 129.8$ (Ar), 130.2 (Ar), 131.4 (Ar), 133.9 (Ar), 134.9 (Ar), 155.5 $(\mathrm{N}-\mathrm{C}=\mathrm{C}-\mathrm{CO}), 166.3(\mathrm{C}=\mathrm{O})$. EIMS $(\mathrm{m} / \mathrm{z}, \%) 323\left(\mathrm{M}^{+}\right.$, 100), $250\left(\mathrm{C}_{16} \mathrm{H}_{12} \mathrm{NO}_{2}^{+}, 90\right), 178\left(\mathrm{C}_{14} \mathrm{H}_{10}^{+}, 99\right), 77\left(\mathrm{C}_{6} \mathrm{H}_{5}^{+}\right.$, 43). HRMS: calcd for $\mathrm{C}_{20} \mathrm{H}_{21} \mathrm{NO}_{3}$ : 323.15214; found: 323.15254; and deoxybenzoin $(\mathbf{1 5}, \mathrm{R}=\mathrm{H})(25.5 \mathrm{mg}, 45 \%)$ as a colourless solid: $\mathrm{mp} 55-56^{\circ} \mathrm{C}$ (lit.: [25] mp 55-56 ${ }^{\circ} \mathrm{C}$ ). IR $\left(\mathrm{KBr}, \mathrm{cm}^{-1}\right): 1686(\mathrm{C}=\mathrm{O}) .{ }^{1} \mathrm{H}$ NMR $\left(400 \mathrm{MHz} \mathrm{CDCl}_{3}\right)$ $\delta 4.29\left(2 \mathrm{H}, \mathrm{s}, \mathrm{CH}_{2}\right), 7.24-8.04(10 \mathrm{H}, \mathrm{m}, \mathrm{Ar}-\mathrm{H})$. After the reaction in a parallel run was completed, glacial $\mathrm{CH}_{3} \mathrm{COOD}$ (3.0 eq.) was added to the reaction followed by $\mathrm{H}_{2} \mathrm{O}(2 \mathrm{~mL})$. The reaction mixture was diluted with $\mathrm{Et}_{2} \mathrm{O}(5 \mathrm{~mL})$ and washed with an aqueous solution of
$1 \mathrm{M} \mathrm{NaHCO}$. The aqueous layer was extracted with $\mathrm{Et}_{2} \mathrm{O}(3 \times 5 \mathrm{~mL})$, the combined organic extracts were dried over anhydrous sodium sulphate, filtered and concentrated. Purification by CC (silica; $\mathrm{Et}_{2} \mathrm{O} / n$-hexane 1:1) afforded mono-deuterated deoxybenzoin $(\mathbf{1 5}, \mathrm{R}=\mathrm{D})(37.5 \mathrm{mg}$, 66\%): ${ }^{1} \mathrm{H}$ NMR (400 MHz $\left.\mathrm{CDCl}_{3}\right) \delta 4.29(1 \mathrm{H}, \mathrm{s}, \mathrm{CHD})$, $7.23-8.06(10 \mathrm{H}, \mathrm{m}, \mathrm{Ar}-\mathrm{H})$.

\section{Reaction of ethyl 2-allyl-5-oxo-2,5-dihydroisoxazole-4- carboxylate (8e)}

To a stirred solution of compound 8e $(50 \mathrm{mg}, 0.254$ $\mathrm{mmol})$ in dry 1,4-dioxane (1 $\mathrm{mL})$ at RT under argon, was added potassium ethoxide $(32 \mathrm{mg}, 0.38 \mathrm{mmol}, 1.5$ eq.) and 18-crown-6 ether (100 mg, $0.38 \mathrm{mmol})$. On completion of the reaction ( $5 \mathrm{~h}$, TLC control: silica; $\mathrm{Et}_{2} \mathrm{O}$ ), the solvent was removed under reduced pressure and the crude purified by cc (silica; $\left.\mathrm{Et}_{2} \mathrm{O}\right)$. N-Allylmalonamic acid ethyl ester (13) was obtained as a white solid (34.7 $\mathrm{mg}, 80 \%)$ : $\mathrm{mp} 40-41^{\circ} \mathrm{C}\left(\mathrm{Et}_{2} \mathrm{O}\right)$. IR $(\mathrm{KBr}) v_{\max }$ $\left(\mathrm{cm}^{-1}\right): 3299(\mathrm{NH}), 1740$ (C=O ester), $1659(\mathrm{C}=\mathrm{O}$ amide). ${ }^{1} \mathrm{H}$ NMR (400 $\left.\mathrm{MHz} \mathrm{CDCl}_{3}\right) \delta 1.22(3 \mathrm{H}, \mathrm{t}, J=7.1$, $\left.\mathrm{OCH}_{2} \mathrm{CH}_{3}\right), 3.26\left(2 \mathrm{H}, \mathrm{s}, \mathrm{COCH}_{2} \mathrm{COOEt}\right), 3.84(2 \mathrm{H}, \mathrm{t}, J=$ $\left.5.6 \mathrm{~Hz}, \mathrm{NH}-\mathrm{CH}_{2}\right), 4.13\left(2 \mathrm{H}, \mathrm{q}, J=7.1 \mathrm{~Hz}, \mathrm{OCH}_{2} \mathrm{CH}_{3}\right)$, $5.07\left(1 \mathrm{H}, \mathrm{dd}, J=1.1,10.3 \mathrm{~Hz}, \mathrm{CH}_{2}=\mathrm{CH}\right), 5.14(1 \mathrm{H}, \mathrm{dd}$, $\left.J=1.3,17.1 \mathrm{~Hz}, \mathrm{CH}_{2}=\mathrm{CH}\right), 5.80\left(1 \mathrm{H}, \mathrm{m}, \mathrm{CH}_{2}=\mathrm{CH}\right)$, $7.29\left(1 \mathrm{H}\right.$, bs, $\mathrm{NH}$ exchange $\left.\mathrm{D}_{2} \mathrm{O}\right) .{ }^{13} \mathrm{C}$ NMR $(100.62$ $\left.\mathrm{MHz} \mathrm{CDCl}_{3}\right) \delta 14.0\left(\mathrm{COOCH}_{2} \mathrm{CH}_{3}\right), 41.3\left(\mathrm{COCH}_{2} \mathrm{COOEt}\right)$, $41.8\left(\mathrm{NHCH}_{2}\right), 61.5\left(\mathrm{COOCH}_{2} \mathrm{CH}_{3}\right), 116.2\left(\mathrm{CH}_{2}=\mathrm{CH}\right)$, $133.7\left(\mathrm{CH}_{2}=\mathrm{CH}\right), 165.0(\mathrm{NHC}=\mathrm{O}), 169.3$ (COOEt). EIMS (m/z, \%): $171\left(\mathrm{M}^{+}, 10\right), 56\left(\mathrm{C}_{3} \mathrm{H}_{6} \mathrm{~N}^{+}, 100\right)$. HRMS: calcd for $\mathrm{C}_{8} \mathrm{H}_{13} \mathrm{NO}_{3}$ : 171.08954; found: 171.08894 .

\section{Reactions of 2-(2'-methylbut-3'-yn-2'-yl)-3,4- diphenylisoxazolin-5-one (8b)}

With 1.5 eq. of EtOK To a stirred solution of compound (8b) $(15 \mathrm{mg}, 0.055 \mathrm{mmol})$ in dry 1,4-dioxane $(0.25 \mathrm{~mL})$, under argon, was added potassium ethoxide (1.5 eq., 7.0 $\mathrm{mg}, 0.083 \mathrm{mmol}$ ) and 18-crown-6 ether $(21.8 \mathrm{mg}, 0.083$ $\mathrm{mmol})$. On completion of the reaction (10 $\mathrm{min}$ ) (TLC control: silica; $\mathrm{Et}_{2} \mathrm{O} / n$-hexane 3:1), the mixture was neutralized with aq. $\mathrm{AcOH} 0.5 \mathrm{~N}$ and the solvent removed under reduced pressure. The crude was diluted with $\mathrm{Et}_{2} \mathrm{O}(5$ $\mathrm{mL}$ ) and washed with destilled $\mathrm{H}_{2} \mathrm{O}(2 \mathrm{~mL})$. The organic layer was dried over anhydrous sodium sulphate, filtered and concentrated under reduced pressure. Purification by $\mathrm{CC}$ (silica; $\mathrm{Et}_{2} \mathrm{O} / n$-hexane 3:1) afforded 4-(3'-methylbut1',2'-dienyl-1' -yl)-3,4-diphenylisoxazolin-5-one (9b) (4.5 $\mathrm{mg}, 30 \%$ ). After $30 \mathrm{~min}$ of reaction, no rearrangement product could be still detected by TLC.

With 0.1 eq. of EtOK Using the previous conditions with potassium ethoxide ( 0.1 eq.) and 18-crown- 6 ether (0.1 eq.), the reaction after 5 days yielded $\mathbf{9 b}(55 \%)$. 
With LDA To a stirred solution of $\mathbf{8 b}$ (1 eq.) in dry 14dioxane, under argon, were added LDA (1.5 eq., from a $1.47 \mathrm{M}$ solution) and 12-crown-4 ether (1.5 eq.). The reaction mixture was stirred for $48 \mathrm{~h}$. TLC control (silica; $\mathrm{Et}_{2} \mathrm{O} / n$-hexane 3:1) showed no reaction and the starting material was recovered yield (5.4 mg, 90\%).

With tert-BuLi To a stirred solution of $\mathbf{8 b}$ (1 eq.) in dry 1,4-dioxane, under argon, were added tert-BuLi (2 eq., from an hexane solution $0.8 \mathrm{M}, 0.066 \mathrm{mmol}$ ) and 12crown- 4 ether (2 eq.). The reaction mixture was stirred for 70 h. TLC control (silica; $\mathrm{Et}_{2} \mathrm{O} / n$-hexane 3:1) showed no reaction and the starting material was recovered (9.5 mg, 95\%).

\section{Endnote}

${ }^{a}$ Web Table (which contains energy profiles, molecular coordinates of intermediates and transition states as well as normal mode animations) is available via the HTML version of the article.

\section{Additional file}

Additional file 1: Table S1. Calculated relative energies for intermediates and transition states relating to Scheme 6.

\section{Competing interests}

The authors declare that they have no competing interest.

\section{Authors' contributions}

AML, HSR and SP contributed equally to this work. LFVP, MJSG and PMCG carried out the experimental work. All authors read and approved the final manuscript.

\section{Acknowledgements}

We thank Fundação para a Ciência e a Tecnologia (FC\&T, Lisbon, Portugal) for financial support from grant PEst-C/EQB/LA 0006/2011 and Project POCTI/QUI/36456. Three of us (M.J.S.G., L.F.V.P., P.M.C.G.) are grateful for the award of research fellowships from FC\&T.

\section{Author details}

${ }^{1}$ Chemistry Department, REQUIMTE/CQFB, FCT, Universidade Nova de Lisboa, 2829-516, Caparica, Portugal. Departamento das Ciências Naturais e Exatas, Escola Superior de Tecnologia da Saúde de Lisboa, Instituto Politécnico de Lisboa, Lisboa, Portugal. ${ }^{3}$ Chemistry Department, Imperial College London, South Kensington Campus, London, UK.

Received: 20 March 2013 Accepted: 6 May 2013

Published: 28 May 2013

\section{References}

1. Nubbemeyer U: Recent advances in charge-accelerated Aza-Claisen rearrangements. Top Curr Chem 2005, 244:149-213. and references cited therein.

2. Weston MH, Nakajima K, Back TG: Tandem conjugate additions and 3-azaCope rearrangements of tertiary allyl amines and cyclic alpha-vinylamines with acetylenic sulfones. Applications to simple and iterative ring expansions leading to medium and large-ring nitrogen heterocycles. J Org Chem 2008, 73:4630-4637. and references cited therein.

3. Nubbemeyer U: Aza-Claisen Rearrangement. In The Claisen Rearrangement: Methods and Applications. Edited by Hiersemann M, Nubbemeyer U. Weinheim: Wiley-VCH; 2007:461
4. Glória PMC, Prabhakar S, Lobo AM: Studies in N-amino-3-aza Cope rearrangements. Tetrahedron Lett 2008, 49:7355-7357.

5. Gomes MJS, Sharma L, Prabhakar S, Lobo AM, Glória PMC: Studies in 3-oxy -assisted 3-aza Cope rearrangements. Chem Commun 2002:746-747.

6. Hastings CJ, Fiedler D, Bergman RG, Raymond KN: Aza Cope Rearrangement of Propargyl Enammonium Cations Catalyzed By a SelfAssembled 'Nanozyme'. J Am Chem Soc 2008, 130:10977-10983.

7. Pinto LFV, Glória PMC, Gomes MJS, Rzepa HS, Prabhakar S, Lobo AM: A dramatic effect of double bond configuration in N-oxy-3-aza Cope rearrangements $\neg-$ a simple synthesis of functionalised allenes. Tetrahedron Lett 2009, 50:3446-3449.

8. Gilbert JC, Cousins KR: Theoretical and experimental studies of the 3-azaClaisen rearrangement. Tetrahedron 1994, 50:10671-10784.

9. Mitsunobu O, Mulder JA, Xiong H, Zificsak CA, Douglas CJ, Hsung RP: Efficient preparations of novel ynamides and allenamides. Tetrahedron 2001, 57:459-466.

10. Makisumi Y: A facile amino-Claisen rearrangement. Tetrahedron Lett 1966, 7:6413-6417.

11. Makisumi Y, Sasatani T: Sigmatropic tautomerism between n-allyl-3isoxazolin-5-ones and 4-allyl-2-isoxazolin-5-ones. Tetrahedron Lett 1969, 10:543-546.

12. Glória PMC, Prabhakar S, Lobo AM, Gomes MJS: SbCl5-wet acetonitrile: a new system for chemoselective O-desilylation. Tetrahedron Lett 2003, 44:8819-8821.

13. Roustan JLA, Houlihan F: Electrophilie du ligande $\eta^{3}$-allylique de complexes $\eta^{3}$-allyl-dicarbonyl-nitrosyl-fer. Can J Chem 1979 57:2790-2791.

14. Woodman DJ, Stonebroker PM: Ketenimine carboxylate isomer protonation. Heterocycles 1977, 7:247-250. and references cited therein.

15. Khalafy J, Marjani AP, Ebrahimbo ARM: Synthesis of new N-benzoxazole and N-benzothiazole derivatives of 3-(4-substituted- phenyl) aminoisoxazol-5(2H)-ones and comparison of their base induced rearrangement. J Braz Chem Soc 2006, 17:570-576. and references cited therein.

16. Emmons WD: The preparation and properties of oxaziranes. J Am Chem Soc 1957, 79:5739-5754.

17. Butler AR, Challis BC, Lobo AM: Hydrolysis of oxaziridines. Part 3. Evidence for both $\mathrm{O}$ - and $\mathrm{N}$-conjugate acid formation with 2,3,3triethyloxaziridine. J Chem Soc Perkin I/ 1979:1035-1038.

18. Perrin DD, Armarego WLF, Perrin DR: Purification of Laboratory Chemicals. London: Pergamon; 1980

19. Barton DHR, Blazejewski J-C, Charpiot B, Finet J-P, Motherwell WB, Papoula MTB, Stanforth SP: Pentavalent organobismuth reagents. Part 3. Phenylation of enols and of enolate and other anions. J Chem Soc Perkin Trans / 1985:2667-2675.

20. Buckle ALJ, McGookin A, Robertson A: Constituents of "Cortex Piscidiae Erythrinae." Part II. The synthesis of O-methylpiscidic acid. J Chem Soc 1954:3981-3986.

21. Howk BW, McElvain SM: The reaction of organic halides with piperidine. II. Certain alpha-bromo-beta-ketonic esters. J Am Chem Soc 1932, 54:282-289.

22. Logemann H, Almirante L, Caprio L: Studien in der heterocyclischen reihe, II. Mitteil.: die synthese von 1.3.5-triazinen und 5-oxy-isoxazol-derivaten. Chem Ber 1954, 87:1175-1179.

23. Kohler EPB, Blatt $\mathrm{AHAH}$ : Diphenyl isoxazolone: a study of the tautomerism of isoxazolones. J Am Chem Soc 1928, 50:504-515.

24. Teobald BJ: The Nicholas reaction: the use of dicobalt hexacarbonylstabilised propargylic cations in synthesis. Tetrahedron 2002, 58:4133-4170.

25. Barton DHR, Bolton M, Magnus PD, Marath KG, Poulton GA, West PJ: Photochemical transformations. Photolysis of thiobenzoic acid O-esters. Photolysis of O-phenethyl thiobenzoates and other thiobenzoates. J Chem Soc Perkin Trans 1973, 1:1574-1579.

doi:10.1186/1752-153X-7-94

Cite this article as: Gomes et al:: $\mathrm{N}$-heteroatom substitution effect in 3aza-cope rearrangements. Chemistry Central Journal 2013 7:94. 